\title{
THE ASYMPTOTICS OF THE $L^{2}$-ANALYTIC TORSION ON CR MANIFOLDS WITH $S^{1}$ ACTION
}

\author{
RUNG-TZUNG HUANG ${ }^{1, a}$ AND GUOKUAN SHAO ${ }^{2, b}$
}

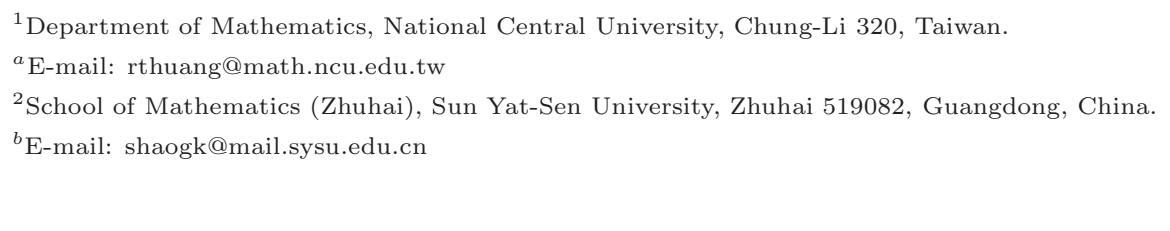

Abstract

Let $X$ be a compact connected CR manifold of dimension $2 n+1, n \geq 1$. Let $\widetilde{X}$ be a paracompact $\mathrm{CR}$ manifold with a transversal CR $S^{1}$-action, such that there is a discrete group $\Gamma$ acting freely on $\widetilde{X}$ having $X=\widetilde{X} / \Gamma$. We introduce the Fourier components of the $L^{2}$-Ray-Singer analytic torsion on $\tilde{X}$ with respect to the $S^{1}$-action. We establish an asymptotic formula for the Fourier components of the $L^{2}$-analytic torsion with respect to the $S^{1}$-action.

\section{Introduction}

In [30], Ray and Singer introduced the holomorphic analytic torsion for $\bar{\partial}$-complex on complex manifolds as the complex analogue of the analytic torsion for flat vector bundles [29]. In [3], Bismut and Vasserot established the asymptotic formula of the holomorphic analytic torsion associated with powers of $p$ of a given positive line bundle over a compact $n$-dimensional complex manifold, as $p \rightarrow+\infty$, by using the heat kernel method of [2] (see also [23, Sect. 5.5]). In [27], M. Puchol extended the results of Bismut and Vasserot on the asymptotic of the holomorphic torsion to the fibration case. Recently, S. Finski [12] studied the asymptotic expansion of holomorphic

Received December 9, 2019.

AMS Subject Classification: Primary: 58J52, 58J28; Secondary: 57Q10.

Key words and phrases: Determinant, Ray-Singer torsion, CR manifolds.

The first author is supported by Taiwan Ministry of Science and Technology project 107-2115-M008-007-MY2. The second author is supported by Sun Yat-Sen University project 74120-18841205. Both authors would like to express their gratitude to Prof. Chin-Yu Hsiao for very helpful comments in this work. 
analytic torsion associated with powers of $p$ of a given positive line bundle over a compact $n$-dimensional complex manifold, as $p \rightarrow+\infty$, and proved that the asymptotic expansion contains only the forms $p^{n-i} \log p, p^{n-i}$ for $i \in \mathbb{N}$. The first two leading terms were proved by Bismut and Vasserot in [3]. The $L^{2}$-torsion have been studied under different assumptions, see [4, 5, 6, 7, 11, 21, 24, 34]. In [32], Su study the asymptotics of the holomorphic $L^{2}$-torsion associated with a power of a positive line bundle.

In orbifold geometry, we have Kawasaki's Hirzebruch-Riemann-Roch formula [20] and also general index theorem [26]. Ma 22] first introduced analytic torsion on orbifolds and obtained an immersion formula for Quillen metrics in the case of orbifolds, which is expressed explicitly in the form of characteristic and secondary characteristic classes on orbifolds. Ma's results should play an important role toward establishing an arithmetic version of the Kawasaki-Riemann-Roch theorem in Arakelov geometry. In [12], S. Finski also calculated the general asymptotic expansion of the analytic torsion for a compact complex orbifold.

Gromov-Henkin-Shubin [14, Theorem 0.2] considered covering manifolds that are strongly pseudoconvex of complex manifolds and analyzed the holomorphic $L^{2}$-functions on the coverings. Todor-Chiose-Marinescu [33] generalized in a similar manner the Morse inequalities of Siu-Demailly [31, 9] on coverings of complex manifolds. The study of problems on CR manifolds with $S^{1}$-action becomes active recently, see [8, 15, 16, 17, 18] and the references therein. In particular, Hsiao-Li [18] established the Morse inequalities for Fourier components of Kohn-Rossi cohomology on $X$ by using the Szegö kernel method. Inspired by the results of [14, 18, 33, 31, 9], the authors establish Morse inequalities for Fourier components of reduced $L^{2}$-Kohn-Rossi cohomology with values in a rigid $\mathrm{CR}$ vector bundle on a covering manifold over a compact connected CR manifold with $S^{1}$-action, see [19]. This generalizes the results of [18] to CR covering manifolds with $S^{1}$-action. We present a proof by the heat kernel method, which is inspired by Bismut's proof [2, 23] of the holomorphic Morse inequalities. The crucial estimate for Fourier components of the heat kernel of Kohn Laplacians was given in [16].

CR geometry is an important subject in several complex variables and is closely related to various research areas. To study further geometric problems for CR manifolds, it is important to know the corresponding heat kernel asymptotics and to have (local) index formula and the concept of analytic 
torsion. The difficulty comes from the fact that the Kohn Laplacian is not hypoelliptic. Thus, we should consider such problems on some class of CR manifolds. It turns out that Kohn's $\square_{b}$ operator on CR manifolds with $S^{1}$ action, including Sasakian manifolds of interest in String Theory (see [25]), is a natural example of geometric significance among those transversally elliptic operators initiated by Atiyah and Singer (see [15], 17], [18] and [8]). In [16], Hsiao-Huang considered a compact connected strongly pseudoconvex CR manifold $X$ and introduced the Fourier components of the Ray-Singer analytic torsion on $X$ with respect to the $S^{1}$-action. They established an asymptotic formula for the Fourier components of the analytic torsion with respect to the $S^{1}$-action. In [12, P. 3501-3502], Finski showed that Theorem 1.5 of [12] gives a refinement of the asymptotic formula of [16]. In this work, we consider a compact connected strongly pseudoconvex CR covering manifold $\widetilde{X}$ and we introduce the Fourier components of the $L^{2}$-Ray-Singer analytic torsion on $\widetilde{X}$ with respect to the $S^{1}$-action. We establish an asymptotic formula for the Fourier components of the $L^{2}$-analytic torsion with respect to the $S^{1}$-action and generalize the results of [16] to CR covering manifolds.

\subsection{Main results}

Now we formulate the main results. We refer to other sections for notations and definitions (see Definition [2.1, 2.2, 2.3, 2.5 and (3.1), (3.22) ) used here. In this work we assume that $X$ is a compact connected strongly pseudoconvex CR manifold of dimension $2 n+1, n \geq 1$ with a transversal CR $S^{1}$-action $e^{i \theta}$ on $X$. For $x \in X$, we say that the period of $x$ is $\frac{2 \pi}{\ell}, \ell \in \mathbb{N}$, if $e^{i \theta} \circ x \neq x$, for every $0<\theta<\frac{2 \pi}{\ell}$, and $e^{i \frac{2 \pi}{\ell}} \circ x=x$. For each $\ell \in \mathbb{N}$, put

and let

$$
X_{\ell}=\left\{x \in X ; \text { the period of } x \text { is } \frac{2 \pi}{\ell}\right\}
$$

$$
p=\min \left\{\ell \in \mathbb{N} ; X_{\ell} \neq \emptyset\right\}
$$

It is well-known that if $X$ is connected, then $X_{p}$ is an open and dense subset of $X$ (see Duistermaat-Heckman [10]). Assume $X=X_{p_{1}} \cup X_{p_{2}} \cup \cdots \cup X_{p_{k}}$, $p=: p_{1}<p_{2}<\cdots<p_{k}$. Set $X_{\text {reg }}:=X_{p}$. We call $x \in X_{\text {reg }}$ a regular point of the $S^{1}$ action. Let $X_{\text {sing }}$ be the complement of $X_{\text {reg }}$. 
Let $\widetilde{X}$ be a paracompact CR manifold, such that there is a discrete group $\Gamma$ acting freely on $\widetilde{X}$ having $X=\widetilde{X} / \Gamma$. Let $\pi: \widetilde{X} \rightarrow X$ be the natural projection with the pull-back map $\pi^{*}: T X \rightarrow T \tilde{X}$. Then $\widetilde{X}$ admits a pull-back CR structure $T^{1,0} \widetilde{X}:=\pi^{*} T^{1,0} X$ and, hence, a CR manifold. We assume that $\widetilde{X}$ admits a transversal CR locally free $S^{1}$ action, denote by $e^{i \theta}$. We further assume that the map

$$
\Gamma \times \widetilde{X} \rightarrow \widetilde{X},(\gamma, \widetilde{x}) \mapsto \gamma \circ \widetilde{x}, \quad \forall \widetilde{x} \in \widetilde{X}, \quad \forall \gamma \in \Gamma .
$$

is $\mathrm{CR}$, see (2.7), and

$$
e^{i \theta} \circ \gamma \circ \widetilde{x}=\gamma \circ e^{i \theta} \circ \widetilde{x}, \quad \forall \widetilde{x} \in \widetilde{X}, \quad \forall \theta \in[0,2 \pi[, \quad \forall \gamma \in \Gamma .
$$

Let $\widetilde{E}:=\pi^{*} E$ be the pull-pack bundle of a rigid CR vector bundle $E$ over $X$. Then $\widetilde{E}$ is a $\Gamma$-invariant rigid $\mathrm{CR}$ vector bundle over $\widetilde{X}$. We denote by $\widetilde{X}_{\text {reg }}$ the set of regular points of the $S^{1}$-action on $\widetilde{X}$. Note that since $\Gamma$ acts on $\widetilde{X}$ freely so that $\widetilde{X} / \Gamma=X$, hence, we have $\widetilde{X}_{\text {reg }} / \Gamma=X_{\text {reg }}=X_{p}$.

Let $N$ be the operator acting on $T^{* 0, q} \widetilde{X}$ by multiplication by $q$. Let $A \in\left(T_{\widetilde{x}}^{* 0, \bullet} \widetilde{X} \otimes \widetilde{E}_{\widetilde{x}}\right) \otimes\left(T_{\widetilde{x}}^{* 0, \bullet} \widetilde{X} \otimes \widetilde{E}_{\widetilde{x}}\right)$, where $\widetilde{E}$ is a rigid CR vector bundle over $\widetilde{X}$. Denote by $\operatorname{STr}_{\Gamma}[A]$ the $\Gamma$-supertrace of $A$ on $\widetilde{X}$, see (4.8). Let

$$
\widetilde{\Pi}_{m}^{\perp}: L_{m}^{2}\left(\widetilde{X}, T^{* 0, \bullet} X \otimes \widetilde{E}\right) \rightarrow\left(\operatorname{Ker} \widetilde{\square}_{b, m}\right)^{\perp}
$$

be the orthogonal projection. In fact, for $\operatorname{Re}(z)>n$, we can define

$$
\widetilde{\theta}_{b, m}(z):=-M\left[\operatorname{STr}_{\Gamma}\left[N e^{-t \widetilde{\square}_{b, m}} \Pi_{m}^{\perp}\right]\right]=-\operatorname{STr}_{\Gamma}\left[N\left(\widetilde{\square}_{b, m}\right)^{-z} \Pi_{m}^{\perp}\right] .
$$

Then we define $\exp \left(-\frac{1}{2} \widetilde{\theta}_{b, m}^{\prime}(0)\right)$ as the $m$ th Fourier component of the analytic torsion on the compact strongly pseudoconvex CR covering manifold $\widetilde{X}$ with a transversal CR $S^{1}$ action. We refer to Section 2 and Section 4 for more details. Our main theorem is the following

Theorem 1.1. With the above notations and assumptions, as $m \rightarrow+\infty$, we have

$$
\begin{aligned}
& \widetilde{\theta}_{b, m}^{\prime}(0)=\frac{p r}{4 \pi} \int_{X} \log \left(\operatorname{det}\left(\frac{m \dot{\mathcal{R}}}{2 \pi}\right)\right) e^{-m \frac{d \omega_{0}}{2 \pi}} \wedge\left(-\omega_{0}\right)+o\left(m^{n}\right), \text { for } p \mid m, \\
& \widetilde{\theta}_{b, m}^{\prime}(0)=o\left(m^{n}\right), \quad \text { for } \quad p \nmid m,
\end{aligned}
$$


where $r$ denotes the rank of $\widetilde{E}$ and $\dot{\mathcal{R}} \in \operatorname{End}\left(T^{1,0} X\right)$ is defined in (3.6).

When $\Gamma=\{e\}$ and $p=1$, we deduce the following result of Hsiao-Huang, see [16, Theorem 5.5]. We denote by $\exp \left(-\frac{1}{2} \theta_{b, m}^{\prime}(0)\right)$ the $m$-th Fourier component of the analytic torsion on the compact strongly pseudoconvex $\mathrm{CR}$ manifold $X$ with a transversal CR $S^{1}$ action, where $\theta_{b, m}(z)$ is defined in (4.14) of [16].

Corollary 1.2. With the above notations and assumptions, as $m \rightarrow+\infty$, we have

$$
\theta_{b, m}^{\prime}(0)=\frac{r}{4 \pi} \int_{X} \log \left(\operatorname{det}\left(\frac{m \dot{\mathcal{R}}}{2 \pi}\right)\right) e^{-m \frac{d \omega_{0}}{2 \pi}} \wedge\left(-\omega_{0}\right)+o\left(m^{n}\right),
$$

where $r$ denotes the rank of $E$ and $\dot{\mathcal{R}} \in \operatorname{End}\left(T^{1,0} X\right)$ is defined in (3.6).

Note that Theorem 1.5 of [12, P. 3501-3502] gives a refinement of Corollary (1.2).

\section{Preliminaries}

\subsection{Some standard notations}

We use the following notations: $\mathbb{N}=\{1,2, \ldots\}, \mathbb{N}_{0}=\mathbb{N} \cup\{0\}, \mathbb{R}$ is the set of real numbers, $\mathbb{R}_{+}:=\{x \in \mathbb{R} ; x>0\}, \overline{\mathbb{R}}_{+}:=\{x \in \mathbb{R} ; x \geq 0\}$. For a multiindex $\alpha=\left(\alpha_{1}, \ldots, \alpha_{n}\right) \in \mathbb{N}_{0}^{n}$ we set $|\alpha|=\alpha_{1}+\cdots+\alpha_{n}$. For $x=$ $\left(x_{1}, \ldots, x_{n}\right)$ we write

$$
x^{\alpha}=x_{1}^{\alpha_{1}} \ldots x_{n}^{\alpha_{n}}, \quad \partial_{x_{j}}=\frac{\partial}{\partial x_{j}}, \quad \partial_{x}^{\alpha}=\partial_{x_{1}}^{\alpha_{1}} \ldots \partial_{x_{n}}^{\alpha_{n}}=\frac{\partial^{|\alpha|}}{\partial x^{\alpha}} .
$$

Let $z=\left(z_{1}, \ldots, z_{n}\right), z_{j}=x_{2 j-1}+i x_{2 j}, j=1, \ldots, n$, be coordinates of $\mathbb{C}^{n}$. We write

$$
\begin{gathered}
z^{\alpha}=z_{1}^{\alpha_{1}} \ldots z_{n}^{\alpha_{n}}, \quad \bar{z}^{\alpha}=\bar{z}_{1}^{\alpha_{1}} \ldots \bar{z}_{n}^{\alpha_{n}} \\
\partial_{z_{j}}=\frac{\partial}{\partial z_{j}}=\frac{1}{2}\left(\frac{\partial}{\partial x_{2 j-1}}-i \frac{\partial}{\partial x_{2 j}}\right), \quad \partial_{\bar{z}_{j}}=\frac{\partial}{\partial \bar{z}_{j}}=\frac{1}{2}\left(\frac{\partial}{\partial x_{2 j-1}}+i \frac{\partial}{\partial x_{2 j}}\right), \\
\partial_{z}^{\alpha}=\partial_{z_{1}}^{\alpha_{1}} \ldots \partial_{z_{n}}^{\alpha_{n}}=\frac{\partial^{|\alpha|}}{\partial z^{\alpha}}, \quad \partial_{\bar{z}}^{\alpha}=\partial_{\bar{z}_{1}}^{\alpha_{1}} \ldots \partial_{\bar{z}_{n}}^{\alpha_{n}}=\frac{\partial^{|\alpha|}}{\partial \bar{z}^{\alpha}} .
\end{gathered}
$$


Let $X$ be a $C^{\infty}$ orientable paracompact manifold. We let $T X$ and $T^{*} X$ denote the tangent bundle of $X$ and the cotangent bundle of $X$, respectively. The complexified tangent bundle of $X$ and the complexified cotangent bundle of $X$ will be denoted by $\mathbb{C} T X$ and $\mathbb{C} T^{*} X$, respectively. We write $\langle\cdot, \cdot\rangle$ to denote the pointwise duality between $T^{*} X$ and $T X$. We extend $\langle\cdot, \cdot\rangle$ bilinearly to $\mathbb{C} T^{*} X \times \mathbb{C} T X$. For $u \in \mathbb{C} T^{*} X, v \in \mathbb{C} T X$, we also write $u(v):=\langle u, v\rangle$.

Let $E$ be a $C^{\infty}$ vector bundle over $X$. The fiber of $E$ at $x \in X$ will be denoted by $E_{x}$. Let $F$ be another vector bundle over $X$. We write $E \otimes F$ to denote the vector bundle over $X \times X$ with fiber over $(x, y) \in X \times X$ consisting of the linear maps from $E_{x}$ to $F_{y}$.

Let $Y \subset X$ be an open set. The spaces of smooth sections of $E$ over $Y$ and distribution sections of $E$ over $Y$ will be denoted by $C^{\infty}(Y, E)$ and $D^{\prime}(Y, E)$, respectively. Let $E^{\prime}(Y, E)$ be the subspace of $D^{\prime}(Y, E)$ whose elements have compact support in $Y$. For $m \in \mathbb{R}$, we let $H^{m}(Y, E)$ denote the Sobolev space of order $m$ of sections of $E$ over $Y$. Put

$$
\begin{aligned}
H_{\mathrm{loc}}^{m}(Y, E) & =\left\{u \in D^{\prime}(Y, E) ; \varphi u \in H^{m}(Y, E), \forall \varphi \in C_{0}^{\infty}(Y)\right\}, \\
H_{\mathrm{comp}}^{m}(Y, E) & =H_{\mathrm{loc}}^{m}(Y, E) \cap E^{\prime}(Y, E) .
\end{aligned}
$$

\subsection{CR geometry}

Let $\left(X, T^{1,0} X\right)$ be a paracompact orientable not necessarily compact CR manifold of dimension $2 n+1, n \geq 1$, where $T^{1,0} X$ is a CR structure of $X$. That is, $T^{1,0} X$ is a subbundle of rank $n$ of the complexified tangent bundle $\mathbb{C} T X$, satisfying $T^{1,0} X \cap T^{0,1} X=\{0\}$, where $T^{0,1} X=\overline{T^{1,0} X}$, and $[\mathcal{V}, \mathcal{V}] \subset \mathcal{V}$, where $\mathcal{V}=C^{\infty}\left(X, T^{1,0} X\right)$. We assume that $X$ admits a $S^{1}$ action: $S^{1} \times X \rightarrow X$. We write $e^{i \theta}$ to denote the $S^{1}$ action. Let $T \in$ $C^{\infty}(X, T X)$ be the global real vector field induced by the $S^{1}$ action given by $(T u)(x)=\left.\frac{\partial}{\partial \theta}\left(u\left(e^{i \theta} \circ x\right)\right)\right|_{\theta=0}, u \in C^{\infty}(X)$.

Definition 2.1. We say that the $S^{1}$ action $e^{i \theta}$ is $\mathrm{CR}$ if $\left[T, C^{\infty}\left(X, T^{1,0} X\right)\right] \subset$ $C^{\infty}\left(X, T^{1,0} X\right)$ and the $S^{1}$ action is transversal if for each $x \in X, \mathbb{C} T(x) \oplus$ $T_{x}^{1,0} X \oplus T_{x}^{0,1} X=\mathbb{C} T_{x} X$. Moreover, we say that the $S^{1}$ action is locally free if $T \neq 0$ everywhere. 
We assume throughout that $\left(X, T^{1,0} X\right)$ is a connected CR manifold with a transversal CR locally free $S^{1}$ action $e^{i \theta}$ and we let $T$ be the global vector field induced by the $S^{1}$ action. Let $\omega_{0} \in C^{\infty}\left(X, T^{*} X\right)$ be the global real (Reeb) one form determined by $\left\langle\omega_{0}, u\right\rangle=0$, for every $u \in T^{1,0} X \oplus T^{0,1} X$ and $\left\langle\omega_{0}, T\right\rangle=-1$. Assume $X=X_{p_{1}} \cup X_{p_{2}} \cup \cdots \cup X_{p_{k}}$ (see (1.1)), $p=$ : $p_{1}<p_{2}<\cdots<p_{k}$. In this work, we assume that $p_{1}=1$ and we denote $X_{\text {reg }}:=X_{p_{1}}=X_{1}$.

Definition 2.2. For $p \in X$, the Levi form $\mathcal{L}_{p}$ is the Hermitian quadratic form on $T_{p}^{1,0} X$ given by $\mathcal{L}_{p}(U, \bar{V})=-\frac{1}{2 i}\left\langle d \omega_{0}(p), U \wedge \bar{V}\right\rangle, U, V \in T_{p}^{1,0} X$.

Definition 2.3. If the Levi form $\mathcal{L}_{p}$ is positive definite, we say that $X$ is strongly pseudoconvex at $p$. If the Levi form is positive definite at every point of $X$, we say that $X$ is strongly pseudoconvex.

Denote by $T^{* 1,0} X$ and $T^{* 0,1} X$ the dual bundles of $T^{1,0} X$ and $T^{0,1} X$, respectively. Define the vector bundle of $(0, q)$ forms by $T^{* 0, q} X=\Lambda^{q}\left(T^{* 0,1} X\right)$. Put $T^{* 0, \bullet} X:=\oplus_{j \in\{0,1, \ldots, n\}} T^{* 0, j} X$. Let $D \subset X$ be an open subset. Let $\Omega^{0, q}(D)$ denote the space of smooth sections of $T^{* 0, q} X$ over $D$ and let $\Omega_{0}^{0, q}(D)$ be the subspace of $\Omega^{0, q}(D)$ whose elements have compact support in $D$. Put

$$
\begin{aligned}
& \Omega^{0, \bullet}(D):=\oplus_{j \in\{0,1, \ldots, n\}} \Omega^{0, j}(D), \\
& \Omega_{0}^{0, \bullet}(D):=\oplus_{j \in\{0,1, \ldots, n\}} \Omega_{0}^{0, j}(D) .
\end{aligned}
$$

Similarly, if $E$ is a vector bundle over $D$, then we let $\Omega^{0, q}(D, E)$ denote the space of smooth sections of $T^{* 0, q} X \otimes E$ over $D$ and let $\Omega_{0}^{0, q}(D, E)$ be the subspace of $\Omega^{0, q}(D, E)$ whose elements have compact support in $D$. Put

$$
\begin{aligned}
& \Omega^{0, \bullet}(D, E):=\oplus_{j \in\{0,1, \ldots, n\}} \Omega^{0, j}(D, E), \\
& \Omega_{0}^{0, \bullet}(D, E):=\oplus_{j \in\{0,1, \ldots, n\}} \Omega_{0}^{0, j}(D, E) .
\end{aligned}
$$

Fix $\left.\theta_{0} \in\right]-\pi, \pi\left[, \theta_{0}\right.$ small. Let

$$
d e^{i \theta_{0}}: \mathbb{C} T_{x} X \rightarrow \mathbb{C} T_{e^{i \theta_{0} x}} X
$$


denote the differential map of $e^{i \theta_{0}}: X \rightarrow X$. By the CR property of the $S^{1}$ action, we can check that

$$
\begin{aligned}
d e^{i \theta_{0}}: T_{x}^{1,0} X & \rightarrow T_{e^{i \theta_{0}} x}^{1,0} X, \\
d e^{i \theta_{0}}: T_{x}^{0,1} X & \rightarrow T_{e^{i \theta_{0}} x}^{0,1} X, \\
d e^{i \theta_{0}}(T(x)) & =T\left(e^{i \theta_{0}} x\right) .
\end{aligned}
$$

Let $\left(e^{i \theta_{0}}\right)^{*}: \Lambda^{r}\left(\mathbb{C} T^{*} X\right) \rightarrow \Lambda^{r}\left(\mathbb{C} T^{*} X\right)$ be the pull-back map by $e^{i \theta_{0}}, r=$ $0,1, \ldots, 2 n+1$. From (2.1), it is easy to see that for every $q=0,1, \ldots, n$,

$$
\left(e^{i \theta_{0}}\right)^{*}: T_{e^{i \theta_{0} x}}^{* 0, q} X \rightarrow T_{x}^{* 0, q} X .
$$

Let $u \in \Omega^{0, q}(X)$. Define

$$
T u:=\left.\frac{\partial}{\partial \theta}\left(\left(e^{i \theta}\right)^{*} u\right)\right|_{\theta=0} \in \Omega^{0, q}(X) .
$$

(See also (3.13).) For every $\theta \in \mathbb{R}$ and every $u \in C^{\infty}\left(X, \Lambda^{r}\left(\mathbb{C} T^{*} X\right)\right)$, we write $u\left(e^{i \theta} \circ x\right):=\left(e^{i \theta}\right)^{*} u(x)$. It is clear that for every $u \in C^{\infty}\left(X, \Lambda^{r}\left(\mathbb{C} T^{*} X\right)\right)$, we have

$$
u(x)=\sum_{m \in \mathbb{Z}} \frac{1}{2 \pi} \int_{-\pi}^{\pi} u\left(e^{i \theta} \circ x\right) e^{-i m \theta} d \theta
$$

Let $\bar{\partial}_{b}: \Omega^{0, q}(X) \rightarrow \Omega^{0, q+1}(X)$ be the tangential Cauchy-Riemann operator. From the CR property of the $S^{1}$ action, it is straightforward to see that (see also (3.14))

$$
T \bar{\partial}_{b}=\bar{\partial}_{b} T \text { on } \Omega^{0, \bullet}(X) .
$$

Definition 2.4. Let $D \subset U$ be an open set. We say that a function $u \in$ $C^{\infty}(D)$ is rigid if $T u=0$. We say that a function $u \in C^{\infty}(X)$ is CauchyRiemann (CR for short) if $\bar{\partial}_{b} u=0$. We call $u$ a rigid CR function if $\bar{\partial}_{b} u=0$ and $T u=0$.

Definition 2.5. Let $F$ be a complex vector bundle over $X$. We say that $F$ is rigid (CR) if $X$ can be covered with open sets $U_{j}$ with trivializing frames $\left\{f_{j}^{1}, f_{j}^{2}, \ldots, f_{j}^{r}\right\}, j=1,2, \ldots$, such that the corresponding transition matrices are rigid (CR). The frames $\left\{f_{j}^{1}, f_{j}^{2}, \ldots, f_{j}^{r}\right\}, j=1,2, \ldots$, are called rigid $(\mathrm{CR})$ frames. 
Definition 2.6. Let $F$ be a complex rigid vector bundle over $X$ and let $\langle\cdot \mid \cdot\rangle_{F}$ be a Hermitian metric on $F$. We say that $\langle\cdot \mid \cdot\rangle_{F}$ is a rigid Hermitian metric if for every rigid local frames $f_{1}, \ldots, f_{r}$ of $F$, we have $T\left\langle f_{j} \mid f_{k}\right\rangle_{F}=0$, for every $j, k=1,2, \ldots, r$.

It is known that there is a rigid Hermitian metric on any rigid vector bundle $F$ (see Theorem 2.10 in [8] and Theorem 10.5 in [15]). Note that Baouendi-Rothschild-Treves [1] proved that $T^{1,0} X$ is a rigid complex vector bundle over $X$.

From now on, let $E$ be a rigid $\mathrm{CR}$ vector bundle over $X$ and we take a rigid Hermitian metric $\langle\cdot \mid \cdot\rangle_{E}$ on $E$ and take a rigid Hermitian metric $\langle\cdot \mid \cdot\rangle$ on $\mathbb{C} T X$ such that $T^{1,0} X \perp T^{0,1} X, T \perp\left(T^{1,0} X \oplus T^{0,1} X\right),\langle T \mid T\rangle=1$. The Hermitian metrics on $\mathbb{C} T X$ and on $E$ induce Hermitian metrics $\langle\cdot \mid \cdot\rangle$ and $\langle\cdot \mid \cdot\rangle_{E}$ on $T^{* 0, \bullet} X$ and $T^{* 0, \bullet} X \otimes E$, respectively. Let $A(x, y) \in\left(T_{y}^{*, \bullet} X \otimes\right.$ $\left.E_{y}\right) \otimes\left(T_{x}^{*, \bullet} X \otimes E_{x}\right)$. We write $|A(x, y)|$ to denote the natural matrix norm of $A(x, y)$ induced by $\langle\cdot \mid \cdot\rangle_{E}$. We denote by $d v_{X}=d v_{X}(x)$ the volume form on $X$ induced by the fixed Hermitian metric $\langle\cdot \mid \cdot\rangle$ on $\mathbb{C} T X$. Then we get natural global $L^{2}$ inner products $(\cdot \mid \cdot)_{E},(\cdot \mid \cdot)$ on $\Omega^{0, \bullet}(X, E)$ and $\Omega^{0, \bullet}(X)$, respectively. We denote by $L^{2}\left(X, T^{* 0, q} X \otimes E\right)$ and $L^{2}\left(X, T^{* 0, q} X\right)$ the completions of $\Omega^{0, q}(X, E)$ and $\Omega^{0, q}(X)$ with respect to $(\cdot \mid \cdot)_{E}$ and $(\cdot \mid \cdot)$, respectively. Similarly, we denote by $L^{2}\left(X, T^{* 0, \bullet} X \otimes E\right)$ and $L^{2}\left(X, T^{* 0, \bullet} X\right)$ the completions of $\Omega^{0, \bullet}(X, E)$ and $\Omega^{0, \bullet}(X)$ with respect to $(\cdot \mid \cdot)_{E}$ and $(\cdot \mid \cdot)$, respectively. We extend $(\cdot \mid \cdot)_{E}$ and $(\cdot \mid \cdot)$ to $L^{2}\left(X, T^{* 0, \bullet} X \otimes E\right)$ and $L^{2}\left(X, T^{* 0, \bullet} X\right)$ in the standard way, respectively. For $f \in L^{2}\left(X, T^{* 0, \bullet} X \otimes E\right)$, we denote $\|f\|_{E}^{2}:=(f \mid f)_{E}$. Similarly, for $f \in L^{2}\left(X, T^{* 0, \bullet} X\right)$, we denote $\|f\|^{2}:=$ $(f \mid f)$.

We also write $\bar{\partial}_{b}$ to denote the tangential Cauchy-Riemann operator acting on forms with values in $E$ :

$$
\bar{\partial}_{b}: \Omega^{0, \bullet}(X, E) \rightarrow \Omega^{0, \bullet}(X, E) .
$$

Since $E$ is rigid, we can also define $T u$ for every $u \in \Omega^{0, q}(X, E)$ and we have

$$
T \bar{\partial}_{b}=\bar{\partial}_{b} T \text { on } \Omega^{0, \bullet}(X, E)
$$


For every $m \in \mathbb{Z}$, let

$$
\begin{aligned}
& \Omega_{m}^{0, q}(X, E):=\left\{u \in \Omega^{0, q}(X, E) ; T u=i m u\right\}, \quad q=0,1,2, \ldots, n, \\
& \Omega_{m}^{0, \bullet}(X, E):=\left\{u \in \Omega^{0, \bullet}(X, E) ; T u=i m u\right\} .
\end{aligned}
$$

For each $m \in \mathbb{Z}$, we denote by $L_{m}^{2}\left(X, T^{* 0, q} X \otimes E\right)$ and $L_{m}^{2}\left(X, T^{* 0, q} X\right)$ the completions of $\Omega_{m}^{0, q}(X, E)$ and $\Omega_{m}^{0, q}(X)$ with respect to $(\cdot \mid \cdot)_{E}$ and $(\cdot \mid \cdot)$, respectively. Similarly, we denote by $L_{m}^{2}\left(X, T^{* 0, \bullet} X \otimes E\right)$ and $L_{m}^{2}\left(X, T^{* 0, \bullet} X\right)$ the completions of $\Omega_{m}^{0, \bullet}(X, E)$ and $\Omega_{m}^{0, \bullet}(X)$ with respect to $(\cdot \mid \cdot)_{E}$ and $(\cdot \mid \cdot)$, respectively.

\subsection{Covering manifolds, Von Neumann dimension}

In this subsection, we recall the background on CR covering manifolds, [19, Section 2]. Let $\left(X, T^{1,0} X\right)$ be a compact CR manifold of dimension $2 n+1, n \geq 1$. Let $\widetilde{X}$ be a paracompact CR manifold, such that there is a discrete group $\Gamma$ acting freely on $\widetilde{X}$ having $X=\widetilde{X} / \Gamma$. Let $\pi: \widetilde{X} \rightarrow X$ be the natural projection with the pull-back map $\pi^{*}: T X \rightarrow T \widetilde{X}$. Then $\widetilde{X}$ admits a pull-back CR structure $T^{1,0} \widetilde{X}:=\pi^{*} T^{1,0} X$ and, hence, a CR manifold. We assume that $\tilde{X}$ admits a transversal CR locally free $S^{1}$ action, denoted by $e^{i \theta}$. We further assume that the map

$$
\Gamma \times \widetilde{X} \rightarrow \widetilde{X},(\gamma, \widetilde{x}) \mapsto \gamma \circ \widetilde{x}, \quad \forall \widetilde{x} \in \widetilde{X}, \quad \forall \gamma \in \Gamma .
$$

is $\mathrm{CR}$, i.e.

$$
\gamma_{*}\left(T_{\widetilde{x}}^{1,0} \widetilde{X}\right) \subseteq T_{\gamma \cdot \widetilde{x}}^{1,0} \widetilde{X}
$$

and

$$
e^{i \theta} \circ \gamma \circ \widetilde{x}=\gamma \circ e^{i \theta} \circ \widetilde{x}, \quad \forall \widetilde{x} \in \widetilde{X}, \quad \forall \theta \in[0,2 \pi[, \quad \forall \gamma \in \Gamma .
$$

It is easy to see that the $S^{1}$-action $e^{i \theta}$ on $\widetilde{X}$ induces a transversal CR locally free $S^{1}$ action, also denoted by $e^{i \theta}$. We denote by $\widetilde{T}:=\pi^{*} T$ the pull-back one form on $\widetilde{X}$, then $T$ is the global real vector field induced by the $S^{1}$-action on $X$. Let $\widetilde{\omega}_{0}:=\pi^{*} \omega_{0}$ be the pull-back one form on $\widetilde{X}$, where $\omega_{0}$ is the global real one form on $X$ as defined in Subsection 2.2. Then, for $\widetilde{p} \in \widetilde{X}$, 
the Levi form $\widetilde{\mathcal{L}}_{\widetilde{p}}$ is the Hermitian quadratic form on $T_{\widetilde{p}}^{1,0} \widetilde{X}$ given by

$$
\widetilde{\mathcal{L}}_{\widetilde{p}}(\widetilde{U}, \overline{\widetilde{V}})=-\frac{1}{2 i}\left\langle d \widetilde{\omega}_{0}(\widetilde{p}), \widetilde{U} \wedge \overline{\widetilde{V}}\right\rangle=-\frac{1}{2 i}\left\langle d \omega_{0}(\pi(\widetilde{p})), \pi_{*} \widetilde{U} \wedge \pi_{*} \overline{\widetilde{V}}\right\rangle,
$$

where $\widetilde{U}, \widetilde{V} \in T_{\widetilde{p}}^{1,0} \widetilde{X}$.

As usual, let $\Omega^{0, q}(\widetilde{X})$ denote the space of smooth sections of $\wedge^{q}\left(T^{* 0,1} \widetilde{X}\right)$. We also denote by $\bar{\partial}_{b}: \Omega^{0, q}(\tilde{X}) \rightarrow \Omega^{0, q+1}(\widetilde{X})$ the tangential Cauchy-Riemann operator. Then $\widetilde{T} \bar{\partial}_{b}=\bar{\partial}_{b} \widetilde{T}$ on $\Omega^{0, \bullet}(\widetilde{X})$. Let $E$ be a rigid $\mathrm{CR}$ vector bundle over $X$, then $\widetilde{E}:=\pi^{*} E$ is a $\Gamma$-invariant rigid CR vector bundle over $\widetilde{X}$. Again let $\Omega^{0, q}(\widetilde{X}, \widetilde{E})$ denote the space of smooth sections of $\wedge^{q}\left(T^{* 0,1} \widetilde{X}\right) \otimes \widetilde{E}$. We again denote by $\bar{\partial}_{b}: \Omega^{0, q}(\widetilde{X}, \widetilde{E}) \rightarrow \Omega^{0, q+1}(\widetilde{X}, \widetilde{E})$ the tangential CauchyRiemann operator. Then again $\widetilde{T} \bar{\partial}_{b}=\bar{\partial}_{b} \widetilde{T}$ on $\Omega^{0, \bullet}(\widetilde{X}, \widetilde{E})$. We denote by $L^{2}\left(\widetilde{X}, T^{* 0, q} \widetilde{X} \otimes \widetilde{E}\right)$ and $L^{2}\left(\widetilde{X}, T^{* 0, q} \widetilde{X}\right)$ the completions of $\Omega^{0, q}(\widetilde{X}, \widetilde{E})$ and $\Omega^{0, q}(\widetilde{X})$ with respect to the corresponding pull-back metrics $(\cdot \mid \cdot)_{\widetilde{E}}$ and $(\cdot \mid \cdot)$. Similarly, we denote by $L^{2}\left(\widetilde{X}, T^{* 0, \bullet} \widetilde{X} \otimes \widetilde{E}\right)$ and $L^{2}\left(\widetilde{X}, T^{* 0, \bullet} \widetilde{X}\right)$ the completions of $\Omega^{0, \bullet}(\widetilde{X}, \widetilde{E})$ and $\Omega^{0, \bullet}(\widetilde{X})$ with respect to the corresponding pull-back metrics $(\cdot \mid \cdot)_{\widetilde{E}}$ and $(\cdot \mid \cdot)$.

As usual, for every $m \in \mathbb{Z}$, let

$$
\begin{aligned}
& \Omega_{m}^{0, q}(\widetilde{X}, \widetilde{E}):=\left\{u \in \Omega^{0, q}(\widetilde{X}, \widetilde{E}) ; \widetilde{T} u=i m u\right\}, \quad q=0,1,2, \ldots, n, \\
& \Omega_{m}^{0, \bullet}(\widetilde{X}, \widetilde{E}):=\left\{u \in \Omega^{0, \bullet}(\widetilde{X}, \widetilde{E}) ; \widetilde{T} u=i m u\right\} .
\end{aligned}
$$

For each $m \in \mathbb{Z}$, we denote by $L_{m}^{2}\left(\widetilde{X}, T^{* 0, q} \widetilde{X} \otimes \widetilde{E}\right)$ and $L_{m}^{2}\left(\widetilde{X}, T^{* 0, q} \widetilde{X}\right)$ the completions of $\Omega_{m}^{0, q}(\widetilde{X}, \widetilde{E})$ and $\Omega_{m}^{0, q}(\widetilde{X})$ with respect to the corresponding pull-back metrics $(\cdot \mid \cdot)_{\widetilde{E}}$ and $(\cdot \mid \cdot)$. Similarly, we denote by $L_{m}^{2}\left(\widetilde{X}, T^{* 0, \bullet} \widetilde{X} \otimes\right.$ $\widetilde{E})$ and $L_{m}^{2}\left(\widetilde{X}, T^{* 0, \bullet} \widetilde{X}\right)$ the completions of $\Omega_{m}^{0, \bullet}(\widetilde{X}, \widetilde{E})$ and $\Omega_{m}^{0, \bullet}(\widetilde{X})$ with respect to the corresponding pull-back metrics $(\cdot \mid \cdot)_{\widetilde{E}}$ and $(\cdot \mid \cdot)$.

Recall that $U \subset \widetilde{X}$ is called a fundamental domain of the action of $\Gamma$ on $\widetilde{X}$ if the following conditions hold:

1. $\tilde{X}=\cup_{\gamma \in \Gamma} \gamma(\bar{U})$,

2. $\gamma_{1}(U) \cap \gamma_{2}(U)=\emptyset \quad$ for $\quad \gamma_{1}, \gamma_{2} \in \Gamma, \gamma_{1} \neq \gamma_{2}$,

3. $\bar{U} \backslash U$ is of measure 0 . 
We can take $U$ to be $S^{1}$-invariant and with the pull-back $S^{1}$-action $e^{i \theta}$. We construct such a fundamental domain in the following: From the discussion in the proof of [8, Theorem 2.11], we can find local trivializations $W_{1}, \cdots, W_{N}$ such that $X=\cup_{j=1}^{N} W_{j}$ and each $W_{j}$ is $S^{1}$-invariant. For each $j$, let $\widetilde{W}_{j} \subset \widetilde{X}$ be an $S^{1}$-invariant open set such that $\pi: \widetilde{W}_{j} \rightarrow W_{j}$ is a diffeomorphism and a CR map with inverse $\phi_{j}: W_{j} \rightarrow \widetilde{W}_{j}$. Define $U_{j}=W_{j} \backslash\left(\cup_{i<j} \bar{W}_{i} \cap W_{j}\right)$. Then $U:=\cup_{j} \phi_{j}\left(U_{j}\right)$ is the fundamental domain we want.

It is easy to see that

$$
L^{2}(\widetilde{X}, \widetilde{E}) \simeq L^{2} \Gamma \otimes L^{2}(U, \widetilde{E}) \simeq L^{2} \Gamma \otimes L^{2}(X, E)
$$

We then have a unitary action of $\Gamma$ by left translations on $L^{2} \Gamma$ by $t_{\gamma} \delta_{\eta}=\delta_{\gamma \eta}$, where $\left\{\delta_{\eta}: \eta \in \Gamma\right\}$ is the orthonormal basis of $L^{2} \Gamma$ formed by the delta functions. It induces a unitary action of $\Gamma$ on $L^{2}(\widetilde{X}, \widetilde{E})$ by $\gamma \mapsto T_{\gamma}=t_{\gamma} \otimes$ Id.

Let us recall the definition of the Von Neumann dimension or $\Gamma$-dimension of a $\Gamma$-module $V \subset L^{2}\left(\widetilde{X}, T^{* 0, q} \widetilde{X} \otimes \widetilde{E}\right)$, see also [23, Definition 3.6.1]. We shall denote by $\mathscr{L}(A)$ the space of bounded operators of the Hilbert space $H$. Let $\mathscr{A} \Gamma \subset \mathscr{L}\left(L^{2} \Gamma\right)$ be the algebra of operators which commute with all left translations and denote the unit element of $\Gamma$ by $e$. We define $\operatorname{Tr}_{\Gamma}[A]:=\left\langle A \delta_{e}, \delta_{e}\right\rangle, A \in \mathscr{A}_{\Gamma}$. Note that a $\Gamma$-module is a left $\Gamma$-invariant subspace $V \subset L^{2} \Gamma$. The orthogonal projection $P_{V}$ on $V$ is in $\mathscr{A}_{\Gamma}$ for a $\Gamma$-module $V$. Set $\operatorname{dim}_{\Gamma} V:=\operatorname{Tr}_{\Gamma}\left[P_{V}\right]$. Now we replace $L^{2} \Gamma$ by $L^{2}\left(\widetilde{X}, T^{* 0, q} \widetilde{X} \otimes \widetilde{E}\right)$. Then to any operator $A \in \mathscr{L}\left(L^{2}\left(\widetilde{X}, T^{* 0, q} \widetilde{X} \otimes \widetilde{E}\right)\right)$, we associate operators $a_{\gamma \eta} \in \mathscr{L}\left(L^{2}\left(U, T^{* 0, q} \widetilde{X} \otimes \widetilde{E}\right)\right)$ such that $a_{\gamma \eta}(f)$ is the projection of $A\left(\delta_{\gamma} \otimes f\right)$ on $\mathbb{C} \delta_{\eta} \otimes L^{2}\left(U, T^{* 0, q} \widetilde{X} \otimes \widetilde{E}\right)$. In addition, if $A \in \mathscr{A}_{\Gamma}$ and $A$ is positive, then $a_{\gamma \eta}=a_{e, \gamma^{-1} \eta}$ and

$$
\operatorname{Tr}_{\Gamma}[A]:=\operatorname{Tr}\left[a_{e e}\right] \geq 0,
$$

is well-defined. The orthogonal projection $P_{V}$ on $V \subset L^{2}\left(\widetilde{X}, T^{* 0, q} \widetilde{X} \otimes \widetilde{E}\right)$ is in $\mathscr{A} \Gamma$ for a $\Gamma$-module $V$.

Definition 2.7. The Von Neumann dimension or $\Gamma$-dimension of a $\Gamma$-module $V$ is defined by

$$
\operatorname{dim}_{\Gamma} V:=\operatorname{Tr}_{\Gamma}\left[P_{V}\right] .
$$




\section{Asymptotic expansion of heat kernels of Kohn Laplacians}

In this section, we recall the definition of heat kernels. Then we recall the asymptotic expansions of heat kernels of Kohn Laplacians on a CR covering manifold, see [19, Section 3].

\subsection{Asymptotics of heat kernels of Kohn Laplacians on a compact CR manifold}

Since $T \bar{\partial}_{b}=\bar{\partial}_{b} T$ and $E$ is a rigid CR vector bundle with a rigid Hermitian metric, we have

$$
\bar{\partial}_{b, m}:=\bar{\partial}_{b}: \Omega_{m}^{0, \bullet}(X, E) \rightarrow \Omega_{m}^{0, \bullet}(X, E), \quad \forall m \in \mathbb{Z} .
$$

The $m$-th Fourier component of Kohn-Rossi cohomology is given by

$$
H_{b, m}^{q}(X, E):=\frac{\operatorname{Ker} \bar{\partial}_{b}: \Omega_{m}^{0, q}(X, E) \rightarrow \Omega_{m}^{0, q+1}(X, E)}{\operatorname{Im} \bar{\partial}_{b}: \Omega_{m}^{0, q-1}(X, E) \rightarrow \Omega_{m}^{0, q}(X, E)} .
$$

We also write

$$
\bar{\partial}_{b}^{*}: \Omega^{0, \bullet}(X, E) \rightarrow \Omega^{0, \bullet}(X, E)
$$

to denote the formal adjoint of $\bar{\partial}_{b}$ with respect to $(\cdot \mid \cdot)_{E}$. Since $\langle\cdot \mid \cdot\rangle_{E}$ and $\langle\cdot \mid \cdot\rangle$ are rigid, we can check that

$$
\begin{aligned}
& T \bar{\partial}_{b}^{*}=\bar{\partial}_{b}^{*} T \text { on } \Omega^{0, \bullet}(X, E), \\
& \bar{\partial}_{b, m}^{*}:=\bar{\partial}_{b}^{*}: \Omega_{m}^{0, \bullet}(X, E) \rightarrow \Omega_{m}^{0, \bullet}(X, E), \quad \forall m \in \mathbb{Z} .
\end{aligned}
$$

Now, we fix $m \in \mathbb{Z}$. The $m$-th Fourier component of Kohn Laplacian is given by

$$
\square_{b, m}:=\left(\bar{\partial}_{b, m}+\bar{\partial}_{b, m}^{*}\right)^{2}: \Omega_{m}^{0, \bullet}(X, E) \rightarrow \Omega_{m}^{0, \bullet}(X, E) .
$$

We extend $\square_{b, m}$ to $L_{m}^{2}\left(X, T^{* 0, \bullet} X \otimes E\right)$ by

$$
\square_{b, m}: \operatorname{Dom} \square_{b, m} \subset L_{m}^{2}\left(X, T^{* 0, \bullet} X \otimes E\right) \rightarrow L_{m}^{2}\left(X, T^{* 0, \bullet} X \otimes E\right),
$$

where Dom $\square_{b, m}:=\left\{u \in L_{m}^{2}\left(X, T^{* 0, \bullet} X \otimes E\right) ; \square_{b, m} u \in L_{m}^{2}\left(X, T^{* 0, \bullet} X \otimes E\right)\right\}$, where for any $u \in L_{m}^{2}\left(X, T^{* 0, \bullet} X \otimes E\right), \square_{b, m} u$ is defined in the sense of distributions. We recall the following results (see Section 3 in [8]). 
Theorem 3.1. The Kohn Laplacian $\square_{b, m}$ is self-adjoint, Spec $\square_{b, m}$ is a discrete subset of $\left[0, \infty\left[\right.\right.$ and for every $\nu \in \operatorname{Spec} \square_{b, m}, \nu$ is an eigenvalue of $\square_{b, m}$ with finite multiplicity.

For every $\nu \in \operatorname{Spec} \square_{b, m}$, let $\left\{f_{1}^{\nu}, \ldots, f_{d_{\nu}}^{\nu}\right\}$ be an orthonormal frame for the eigenspace of $\square_{b, m}$ with eigenvalue $\nu$. The heat kernel $e^{-t \square_{b, m}}(x, y)$ is given by

$$
e^{-t \square_{b, m}}(x, y)=\sum_{\nu \in \operatorname{Spec} \square_{b, m}} \sum_{j=1}^{d_{\nu}} e^{-\nu t} f_{j}^{\nu}(x) \otimes\left(f_{j}^{\nu}(y)\right)^{\dagger},
$$

where $f_{j}^{\nu}(x) \otimes\left(f_{j}^{\nu}(y)\right)^{\dagger}$ denotes the linear map:

$$
\begin{aligned}
& f_{j}^{\nu}(x) \otimes\left(f_{j}^{\nu}(y)\right)^{\dagger}: T_{y}^{* 0, \bullet} X \otimes E_{y} \rightarrow T_{x}^{* 0, \bullet} X \otimes E_{x}, \\
& u(y) \in T_{y}^{* 0, \bullet} X \otimes E_{y} \rightarrow f_{j}^{\nu}(x)\left\langle u(y) \mid f_{j}^{\nu}(y)\right\rangle_{E} \in T_{x}^{* 0, \bullet} X \otimes E_{x} .
\end{aligned}
$$

Let $e^{-t \square_{b, m}}: L^{2}\left(X, T^{* 0, \bullet} X \otimes E\right) \rightarrow L_{m}^{2}\left(X, T^{* 0, \bullet} X \otimes E\right)$ be the continuous operator with distribution kernel $e^{-t \square_{b, m}}(x, y)$.

We denote by $\dot{\mathcal{R}}$ the Hermitian matrix $\dot{\mathcal{R}} \in \operatorname{End}\left(T^{1,0} X\right)$ such that for $V, W \in T^{1,0} X$

$$
i d \omega_{0}(V, \bar{W})=\langle\dot{\mathcal{R}} V \mid W\rangle .
$$

Let $\left\{\omega_{j}\right\}_{j=1}^{n}$ be a local orthonormal frame of $T^{1,0} X$ with dual frame $\left\{\omega^{j}\right\}_{j=1}^{n}$. Set

$$
\gamma_{d}=-i \sum_{l, j=1}^{n} d \omega_{0}\left(\omega_{j}, \bar{\omega}_{l}\right) \bar{\omega}^{l} \wedge \iota \bar{\omega}_{j}
$$

where $\iota_{\bar{\omega}_{j}}$ denotes the interior product of $\bar{\omega}_{j}$. Then $\gamma_{d} \in \operatorname{End}\left(T^{* 0, \bullet} X\right)$ and $-i d \omega_{0}$ acts as the derivative $\gamma_{d}$ on $T^{* 0, \bullet} X$. If we choose $\left\{\omega_{j}\right\}_{j=1}^{n}$ to be an orthonormal basis of $T^{1,0} X$ such that

$$
\dot{\mathcal{R}}(x)=\operatorname{diag}\left(a_{1}(x), \cdots, a_{n}(x)\right) \in \operatorname{End}\left(T_{x}^{1,0} X\right),
$$

then

$$
\gamma_{d}(x)=-\sum_{j=1}^{n} a_{j}(x) \bar{\omega}^{j} \wedge \iota_{\bar{\omega}_{j}} .
$$

Define $\operatorname{det} \dot{\mathcal{R}}(x):=a_{1}(x) \cdots a_{n}(x)$. 
Fix $x, y \in X$. Let $d(x, y)$ denote the standard Riemannian distance of $x$ and $y$ with respect to the given Hermitian metric. Take $\zeta$

$$
o<\zeta<\inf \left\{\frac{2 \pi}{p_{k}},\left|\frac{2 \pi}{p_{r}}-\frac{2 \pi}{p_{r+1}}\right|, r=1, \cdots, k-1\right\} .
$$

For $x \in X$, put

$$
\hat{d}\left(x, X_{\text {sing }}\right):=\inf \left\{d\left(x, e^{-i \theta} x\right) ; \zeta \leq \theta \leq \frac{2 \pi}{p}-\zeta\right\} .
$$

The following result generalizes Theorem 3.1 in [16], see [19, Theorem $3.2]$.

Theorem 3.2. With the above notations and assumptions, for every $\epsilon>0$, there are $m_{0}>0, \varepsilon_{0}>0$ and $C>0$ such that for all $m \geq m_{0}$, we have

$$
\begin{aligned}
& \left|e^{-\frac{t}{m} \square_{b, m}}(x, x)-\sum_{s=1}^{p} e^{\frac{2 \pi(s-1)}{p} m i}(2 \pi)^{-n-1} m^{n} \frac{\operatorname{det}(\dot{\mathcal{R}}) \exp \left(t \gamma_{d}\right)}{\operatorname{det}(1-\exp (-t \dot{\mathcal{R}}))}(x) \otimes \operatorname{Id}_{E_{x}}\right| \\
& \leq \epsilon m^{n}+C m^{n} t^{-n} e^{\frac{-\varepsilon_{0} m \hat{d}\left(x, X_{\text {sing }}\right)^{2}}{t}}, \quad \forall(t, x) \in \mathbb{R}_{+} \times X_{\text {reg }} .
\end{aligned}
$$

\subsection{BRT trivializations}

To prove Theorem 3.2, we need some preparations. We first need the following result due to Baouendi-Rothschild-Treves [1].

Theorem 3.3. For every point $x_{0} \in X$, we can find local coordinates $x=$ $\left(x_{1}, \ldots, x_{2 n+1}\right)=(z, \theta)=\left(z_{1}, \ldots, z_{n}, \theta\right), z_{j}=x_{2 j-1}+i x_{2 j}, j=1, \ldots, n$, $x_{2 n+1}=\theta$, defined in some small neighborhood $D=\left\{(z, \theta):|z|<\delta,-\varepsilon_{0}<\right.$ $\left.\theta<\varepsilon_{0}\right\}$ of $x_{0}, \delta>0,0<\varepsilon_{0}<\pi$, such that $\left(z\left(x_{0}\right), \theta\left(x_{0}\right)\right)=(0,0)$ and

$$
\begin{aligned}
& T=\frac{\partial}{\partial \theta} \\
& Z_{j}=\frac{\partial}{\partial z_{j}}+i \frac{\partial \varphi}{\partial z_{j}}(z) \frac{\partial}{\partial \theta}, j=1, \ldots, n
\end{aligned}
$$

where $Z_{j}(x), j=1, \ldots, n$, form a basis of $T_{x}^{1,0} X$, for each $x \in D$, and $\varphi(z) \in$ $C^{\infty}(D, \mathbb{R})$ is independent of $\theta$. We call $(D,(z, \theta), \varphi)$ BRT trivialization. 
By using BRT trivialization, we get another way to define $T u, \forall u \in$ $\Omega^{0, q}(X)$. Let $(D,(z, \theta), \varphi)$ be a BRT trivialization. It is clear that

$$
\left\{d \overline{z_{j_{1}}} \wedge \cdots \wedge d \overline{z_{j_{q}}}, 1 \leq j_{1}<\cdots<j_{q} \leq n\right\}
$$

is a basis for $T_{x}^{* 0, q} X$, for every $x \in D$. Let $u \in \Omega^{0, q}(X)$. On $D$, we write

$$
u=\sum_{1 \leq j_{1}<\cdots<j_{q} \leq n} u_{j_{1} \cdots j_{q}} d \overline{z_{j_{1}}} \wedge \cdots \wedge d \overline{z_{j_{q}}}
$$

Then, on $D$, we can check that

$$
T u=\sum_{1 \leq j_{1}<\cdots<j_{q} \leq n}\left(T u_{j_{1} \cdots j_{q}}\right) d \overline{z_{j_{1}}} \wedge \cdots \wedge d \overline{z_{j_{q}}}
$$

and $T u$ is independent of the choice of BRT trivializations. Note that, on BRT trivialization $(D,(z, \theta), \varphi)$, we have

$$
\bar{\partial}_{b}=\sum_{j=1}^{n} d \bar{z}_{j} \wedge\left(\frac{\partial}{\partial \bar{z}_{j}}-i \frac{\partial \varphi}{\partial \bar{z}_{j}}(z) \frac{\partial}{\partial \theta}\right) .
$$

\subsection{Local heat kernels on BRT trivializations}

Until further notice, we fix $m \in \mathbb{Z}$. Let $B:=(D,(z, \theta), \varphi)$ be a BRT trivialization. We may assume that $D=U \times]-\varepsilon, \varepsilon[$, where $\varepsilon>0$ and $U$ is an open set of $\mathbb{C}^{n}$. Since $E$ is rigid, we can consider $E$ as a holomorphic vector bundle over $U$. We may assume that $E$ is trivial on $U$. Consider a trivial line bundle $L \rightarrow U$ with non-trivial Hermitian fiber metric $|1|_{h^{L}}^{2}=e^{-2 \varphi}$. Let $\left(L^{m}, h^{L^{m}}\right) \rightarrow U$ be the $m$-th power of $\left(L, h^{L}\right)$. Let $\Omega^{0, q}\left(U, E \otimes L^{m}\right)$ and $\Omega^{0, q}(U, E)$ be the spaces of $(0, q)$ forms on $U$ with values in $E \otimes L^{m}$ and $E$, respectively, $q=0,1,2, \ldots, n$. Put

$$
\begin{aligned}
& \Omega^{0, \bullet}\left(U, E \otimes L^{m}\right):=\oplus_{j \in\{0,1, \ldots, n\}} \Omega^{0, j}\left(U, E \otimes L^{m}\right), \\
& \Omega^{0, \bullet}(U, E):=\oplus_{j \in\{0,1, \ldots, n\}} \Omega^{0, j}(U, E) .
\end{aligned}
$$

Since $L$ is trivial, from now on, we identify $\Omega^{0, \bullet}(U, E)$ with $\Omega^{0, \bullet}\left(U, E \otimes L^{m}\right)$. Since the Hermitian fiber metric $\langle\cdot \mid \cdot\rangle_{E}$ is rigid, we can consider $\langle\cdot \mid \cdot\rangle_{E}$ as a Hermitian fiber metric on the holomorphic vector bundle $E$ over $U$. Let 
$\langle\cdot, \cdot\rangle$ be the Hermitian metric on $\mathbb{C} T U$ given by

$$
\left\langle\frac{\partial}{\partial z_{j}}, \frac{\partial}{\partial z_{k}}\right\rangle=\left\langle\frac{\partial}{\partial z_{j}}+i \frac{\partial \varphi}{\partial z_{j}}(z) \frac{\partial}{\partial \theta} \mid \frac{\partial}{\partial z_{k}}+i \frac{\partial \varphi}{\partial z_{k}}(z) \frac{\partial}{\partial \theta}\right\rangle, \quad j, k=1,2, \ldots, n .
$$

$\langle\cdot, \cdot\rangle$ induces a Hermitian metric on $T^{* 0, \bullet} U:=\oplus_{j=0}^{n} T^{* 0, j} U$, where $T^{* 0, j} U$ is the bundle of $(0, j)$ forms on $U, j=0,1, \ldots, n$. We shall also denote this induced Hermitian metric on $T^{* 0, \bullet} U$ by $\langle\cdot, \cdot\rangle$. The Hermitian metrics on $T^{* 0, \bullet} U$ and $E$ induce a Hermitian metric on $T^{* 0, \bullet} U \otimes E$. We shall also denote this induced metric by $\langle\cdot \mid \cdot\rangle_{E}$. Let $(\cdot, \cdot)$ be the $L^{2}$ inner product on $\Omega^{0, \bullet}(U, E)$ induced by $\langle\cdot, \cdot\rangle,\langle\cdot \mid \cdot\rangle_{E}$. Similarly, let $(\cdot, \cdot)_{m}$ be the $L^{2}$ inner product on $\Omega^{0, \bullet}\left(U, E \otimes L^{m}\right)$ induced by $\langle\cdot, \cdot\rangle,\langle\cdot \mid \cdot\rangle_{E}$ and $h^{L^{m}}$.

The curvature of $L$ induced by $h^{L}$ is given by $R^{L}:=2 \partial \bar{\partial} \varphi$. Let $\dot{R}^{L} \in$ $\operatorname{End}\left(T^{1,0} U\right)$ be the Hermitian matrix given by

$$
R^{L}(W, \bar{Y})=\left\langle\dot{R}^{L} W, Y\right\rangle, \quad W, Y \in T^{1,0} U .
$$

Let $\left\{w_{j}\right\}_{j=1}^{n}$ be a local orthonormal frame of $T^{1,0} U$ with dual frame $\left\{w^{j}\right\}_{j=1}^{n}$. Set

$$
\omega_{d}=-\sum_{l, j} R^{L}\left(w_{j}, \bar{w}_{l}\right) \bar{w}^{l} \wedge \iota \bar{w}_{j},
$$

where $\iota \bar{w}_{j}$ denotes the interior product of $\bar{w}_{j}$.

Let

$$
\bar{\partial}: \Omega^{0, \bullet}\left(U, E \otimes L^{m}\right) \rightarrow \Omega^{0, \bullet}\left(U, E \otimes L^{m}\right)
$$

be the Cauchy-Riemann operator and let

$$
\bar{\partial}^{*, m}: \Omega^{0, \bullet}\left(U, E \otimes L^{m}\right) \rightarrow \Omega^{0, \bullet}\left(U, E \otimes L^{m}\right)
$$

be the formal adjoint of $\bar{\partial}$ with respect to $(\cdot, \cdot)_{m}$. Put

$$
\square_{B, m}:=\left(\bar{\partial}+\bar{\partial}^{*, m}\right)^{2}: \Omega^{0, \bullet}\left(U, E \otimes L^{m}\right) \rightarrow \Omega^{0, \bullet}\left(U, E \otimes L^{m}\right) .
$$

We need the following result (see Lemma 5.1 in [8])

Lemma 3.4. Let $u \in \Omega_{m}^{0, \bullet}(X, E)$. On $D$, we write $u(z, \theta)=e^{i m \theta} \widetilde{u}(z)$, $\widetilde{u}(z) \in \Omega^{0, \bullet}(U, E)$. Then,

$$
e^{-m \varphi} \square_{B, m}\left(e^{m \varphi} \widetilde{u}\right)=e^{-i m \theta} \square_{b, m}(u) .
$$


Let $z, w \in U$ and let $T(z, w) \in\left(T_{w}^{* 0, \bullet} U \otimes E_{w}\right) \otimes\left(T_{z}^{* 0, \bullet} U \otimes E_{z}\right)$. We write $|T(z, w)|$ to denote the standard pointwise matrix norm of $T(z, w)$ induced by $\langle\cdot \mid \cdot\rangle_{E}$. Let $\Omega_{0}^{0, \bullet}(U, E)$ be the subspace of $\Omega^{0, \bullet}(U, E)$ whose elements have compact support in $U$. Let $d v_{U}$ be the volume form on $U$ induced by $\langle\cdot, \cdot\rangle$. Assume $T(z, w) \in C^{\infty}\left(U \times U,\left(T_{w}^{* 0, \bullet} U \otimes E_{w}\right) \otimes\left(T_{z}^{* 0, \bullet} U \otimes E_{z}\right)\right)$. Let $u \in \Omega_{0}^{0, \bullet}(U, E)$. We define the integral $\int T(z, w) u(w) d v_{U}(w)$ in the standard way. Let $G(t, z, w) \in C^{\infty}\left(\mathbb{R}_{+} \times U \times U,\left(T_{w}^{* 0, \bullet} U \otimes E_{w}\right) \otimes\left(T_{z}^{* 0, \bullet} U \otimes E_{z}\right)\right)$. We write $G(t)$ to denote the continuous operator

$$
\begin{aligned}
G(t): \Omega_{0}^{0, \bullet}(U, E) & \rightarrow \Omega^{0, \bullet}(U, E), \\
u & \rightarrow \int G(t, z, w) u(w) d v_{U}(w)
\end{aligned}
$$

and we write $G^{\prime}(t)$ to denote the continuous operator

$$
\begin{aligned}
G^{\prime}(t): \Omega_{0}^{0, \bullet}(U, E) & \rightarrow \Omega^{0, \bullet}(U, E), \\
u & \rightarrow \int \frac{\partial G(t, z, w)}{\partial t} u(w) d v_{U}(w) .
\end{aligned}
$$

We consider the heat operator of $\square_{B, m}$. By using the standard Dirichlet heat kernel construction (see [13]) and the proofs of Theorem 1.6.1 and Theorem 5.5.9 in [23], we deduce the following

Theorem 3.5. There is $A_{B, m}(t, z, w) \in C^{\infty}\left(\mathbb{R}_{+} \times U \times U,\left(T_{w}^{* 0, \bullet} U \otimes E_{w}\right) \otimes\right.$ $\left.\left(T_{z}^{* 0, \bullet} U \otimes E_{z}\right)\right)$ such that

$$
\begin{aligned}
& \lim _{t \rightarrow 0+} A_{B, m}(t)=I \text { in } D^{\prime}\left(U, T^{* 0, \bullet} U \otimes E\right), \\
& A_{B, m}^{\prime}(t) u+\frac{1}{m} A_{B, m}(t)\left(\square_{B, m} u\right)=0, \forall u \in \Omega_{0}^{0, \bullet}(U, E), \forall t>0,
\end{aligned}
$$

and $A_{B, m}(t, z, w)$ satisfies the following:

(I) For every compact set $K \Subset U, \alpha_{1}, \alpha_{2}, \beta_{1}, \beta_{2} \in \mathbb{N}_{0}^{n}$, there are constants $C_{\alpha_{1}, \alpha_{2}, \beta_{1}, \beta_{2}, K}>0$ and $\varepsilon_{0}>0$ independent of $t$ and $m$ such that

$$
\begin{aligned}
& \left|\partial_{z}^{\alpha_{1}} \partial_{\bar{z}}^{\alpha_{2}} \partial_{w}^{\beta_{1}} \partial_{\bar{w}}^{\beta_{2}}\left(A_{B, m}(t, z, w) e^{m(\varphi(w)-\varphi(z))}\right)\right| \\
& \leq C_{\alpha_{1}, \alpha_{2}, \beta_{1}, \beta_{2}, K}\left(\frac{m}{t}\right)^{n+\left|\alpha_{1}\right|+\left|\alpha_{2}\right|+\left|\beta_{1}\right|+\left|\beta_{2}\right|} e^{-m \varepsilon_{0} \frac{|z-w|^{2}}{t}}, \forall(t, z, w) \in \mathbb{R}_{+} \times K \times K .
\end{aligned}
$$


(II) $A_{B, m}(t, z, z)$ admits an asymptotic expansion:

$$
A_{B, m}(t, z, z)=(2 \pi)^{-n} m^{n} \frac{\operatorname{det}\left(\dot{R}^{L}\right) \exp \left(t \omega_{d}\right)}{\operatorname{det}\left(1-\exp \left(-t \dot{R}^{L}\right)\right)}(z) \otimes \operatorname{Id}_{E_{z}}+o\left(m^{n}\right)
$$

in $C^{\ell}\left(U, \operatorname{End}\left(T^{* 0, \bullet} U\right) \otimes E\right)$ locally uniformly on $\mathbb{R}_{+} \times U$, for every $\ell \in \mathbb{N}$. Here we use the convention that if an eigenvalue $a_{j}(z)$ of $\dot{R}^{L}(z)$ is zero, then its contribution for $\frac{\operatorname{det}\left(\dot{R}^{L}\right)}{\operatorname{det}\left(1-\exp \left(-t \dot{R}^{L}\right)\right)}(z)$ is $\frac{1}{t}$.

\section{4. $L^{2}$ Kohn-Rossi cohomology on a covering manifold}

Let

$$
\widetilde{\square}_{b}: \operatorname{Dom} \widetilde{\square}_{b} \subset L^{2}\left(\widetilde{X}, T^{* 0, \bullet} \widetilde{X}\right) \rightarrow L^{2}\left(\widetilde{X}, T^{* 0, \bullet} \widetilde{X}\right)
$$

be the Gaffney extension of the pull-back Kohn Laplacian on $\widetilde{X}$. By a result of Gaffney, $\widetilde{\square}_{b}$ is a positive self-adjoint operator (see Proposition 3.1.2 in Ma-Marinescu 23] ). That is, $\widetilde{\square}_{b}$ is self-adjoint and the spectrum of $\widetilde{\square}_{b}$ is contained in $\overline{\mathbb{R}}_{+}$. Now, we fix $m \in \mathbb{Z}$. As in (3.3), we introduce the $m$-th Fourier component of the Kohn Laplacian $\widetilde{\square}_{b, m}$ on $\Omega_{m}^{0, \bullet}(\widetilde{X}, \widetilde{E})$. We can easily see that $\widetilde{\square}_{b, m}$ is also self-adjoint. By the second isomorphism of (2.11), we can see that, for any $\gamma \in \Gamma$,

$$
T_{\gamma}\left(\operatorname{Dom}\left(\widetilde{\square}_{b, m}\right)\right) \subset \operatorname{Dom}\left(\widetilde{\square}_{b, m}\right), T_{\gamma} \widetilde{\square}_{b, m}=\widetilde{\square}_{b, m} T_{\gamma} \text { on } \operatorname{Dom}\left(\widetilde{\square}_{b, m}\right) \text {. }
$$

Consider the spectral resolution $E_{\lambda}^{q}\left(\widetilde{\square}_{b, m}\right)$ of $\widetilde{\square}_{b, m}$ acting on $L_{m}^{2}\left(\widetilde{X}, T^{* 0, q} \widetilde{X} \otimes \widetilde{E}\right)$. (See [23, Appendix C.2]). The proof of the following lemma is similar to Lemma 3.6.3 in Ma-Marinescu [23], see [19, Lemma 3.7].

Lemma 3.6. For any $q=0,1, \cdots, n$ and $\lambda \in \mathbb{R}$, then $E_{\lambda}^{q}\left(\widetilde{\square}_{b, m}\right)$ commutes with $\Gamma$, its Schwartz kernel is smooth and

$$
\operatorname{dim}_{\Gamma} E_{\lambda}^{q}\left(\widetilde{\square}_{b, m}\right)<+\infty
$$

\section{Definition 3.7.}

(a) The $m$-th Fourier component of the space of harmonic forms $\mathcal{H}^{\bullet}(\widetilde{X}, \widetilde{E})$ is defined by

$$
\mathcal{H}_{b, m}^{\bullet}(\widetilde{X}, \widetilde{E}):=\operatorname{Ker}\left(\widetilde{\square}_{b, m}\right)=\left\{s \in \operatorname{Dom} \widetilde{\square}_{b, m}: \widetilde{\square}_{b, m} s=0\right\} .
$$


(b) The $m$-th Fourier component of the $q$-th reduced $L^{2}$ Kohn-Rossi cohomology is given by

$$
\bar{H}_{b,(2), m}^{q}(\widetilde{X}, \widetilde{E}):=\frac{\operatorname{Ker} \bar{\partial}_{b} \cap L_{m}^{2}\left(\widetilde{X}, T^{* 0, q} \widetilde{X} \otimes \widetilde{E}\right)}{\left[\operatorname{Im} \bar{\partial}_{b} \cap L_{m}^{2}\left(\widetilde{X}, T^{* 0, q} \widetilde{X} \otimes \widetilde{E}\right)\right]},
$$

where $[V]$ denotes the closure of the space $V$.

We can easily obtain the following weak Hodge decomposition

$$
L_{m}^{2}\left(\widetilde{X}, T^{* 0, \bullet} \widetilde{X} \otimes \widetilde{E}\right)=\mathcal{H}^{\bullet}(\widetilde{X}, \widetilde{E}) \oplus\left[\operatorname{Im}\left(\bar{\partial}_{b, m}\right)\right] \oplus\left[\operatorname{Im}\left(\bar{\partial}_{b, m}^{*}\right)\right]
$$

By (3.23), we the the isomorphism

$$
\bar{H}_{b,(2), m}^{\bullet}(\widetilde{X}, \widetilde{E}) \cong \mathcal{H}_{b}^{\bullet}(\widetilde{X}, \widetilde{E}) .
$$

\subsection{Asymptotics of heat kernels of Kohn Laplacians on a covering manifold}

Assume that $X=D_{1} \bigcup D_{2} \bigcup \cdots \cup D_{N}$, where $B_{j}:=\left(D_{j},(z, \theta), \varphi_{j}\right)$ is a BRT trivialization, for each $j$. We may assume that, for each $j, D_{j}=$ $\left.U_{j} \times\right]-2 \delta_{j}, 2 \widetilde{\delta}_{j}\left[\subset \mathbb{C}^{n} \times \mathbb{R}, \delta_{j}>0, \widetilde{\delta}_{j}>0, U_{j}=\left\{z \in \mathbb{C}^{n} ;|z|<l_{j}\right\}\right.$. For each $j$, put $\left.\hat{D}_{j}=\hat{U}_{j} \times\right]-\frac{\delta_{j}}{2}, \frac{\widetilde{\delta}_{j}}{2}\left[\right.$, where $\hat{U}_{j}=\left\{z \in \mathbb{C}^{n} ;|z|<\frac{l_{j}}{2}\right\}$. We may suppose that $X=\hat{D}_{1} \cup \hat{D}_{2} \cup \cdots \cup \hat{D}_{N}$.

Let $\left\{\psi_{j}\right\}$ be a partition of unity subordinate to $\left\{\hat{D}_{j}\right\}$. Then $\left\{\widetilde{\psi}_{\gamma, j}:=\right.$ $\left.\psi_{i} \circ \pi\right\}$ is a partition of unity subordinate to $\left\{\widetilde{D}_{\gamma, j}\right\}$, where $\pi^{-1}\left(\hat{D}_{j}\right)=$ $\cup_{\gamma \in \Gamma} \widetilde{D}_{\gamma, j}$ and $\widetilde{D}_{\gamma_{1}, j}$ and $\widetilde{D}_{\gamma_{2}, j}$ are disjoint for $\gamma_{1} \neq \gamma_{2}$. For each $\gamma \in \Gamma$ and each $j$, we have $\left.\widetilde{D}_{\gamma, j}=\widetilde{U}_{\gamma, j} \times\right]-\frac{\delta_{\gamma, j}}{2}, \frac{\widetilde{\delta}_{\gamma, j}}{2}\left[\right.$, where $\widetilde{U}_{\gamma, j}=\left\{z \in \mathbb{C}^{n} ;|z|<\frac{l_{\gamma, j}}{2}\right\}$. Then $\tilde{X}=\bigcup_{\gamma \in \Gamma} \bigcup_{j=1}^{N} \widetilde{D}_{\gamma, j}$.

Fix $\gamma \in \Gamma$ and $j=1,2, \ldots, N$. Put

$$
K_{\gamma, j}=\left\{z \in \widetilde{U}_{\gamma, j} \text {; there is a } \theta \in\right]-\frac{\delta_{\gamma, j}}{2}, \frac{\widetilde{\delta}_{\gamma, j}}{2}\left[\text { such that } \widetilde{\psi}_{\gamma, j}(z, \theta) \neq 0\right\} .
$$

Let $\tau_{\gamma, j}(z) \in C_{0}^{\infty}\left(\widetilde{U}_{\gamma, j}\right)$ with $\tau_{\gamma, j} \equiv 1$ on some neighborhood $W_{\gamma, j}$ of $K_{\gamma, j}$. Let $\sigma_{\gamma, j} \in C_{0}^{\infty}(]-\frac{\delta_{\gamma, j}}{2}, \frac{\widetilde{\delta}_{\gamma, j}}{2}[)$ with $\int \sigma_{\gamma, j}(\theta) d \theta=1$. Let $\widetilde{A}_{B_{\gamma, j}, m}(t, z, w) \in$ 
$C^{\infty}\left(\mathbb{R}_{+} \times \widetilde{U}_{\gamma, j} \times \widetilde{U}_{\gamma, j},\left(T_{w}^{* 0, \bullet} \widetilde{U}_{\gamma, j} \otimes \widetilde{E}_{w}\right) \otimes\left(T_{z}^{* 0, \bullet} \widetilde{U}_{\gamma, j} \otimes \widetilde{E}_{z}\right)\right)$ be as in Theorem 3.5.

Put

$$
\begin{aligned}
& \widetilde{H}_{\gamma, j, m}(t, \widetilde{x}, \widetilde{y}) \\
& \quad=\widetilde{\psi}_{\gamma, j}(\widetilde{x}) e^{-m \varphi_{j}(z)+i m \theta} \widetilde{A}_{B_{\gamma, j}, m}(t, z, w) e^{m \varphi_{\gamma, j}(w)-i m \eta} \tau_{\gamma, j}(w) \sigma_{\gamma, j}(\eta),
\end{aligned}
$$

where $\widetilde{x}=(z, \theta), \widetilde{y}=(w, \eta) \in \mathbb{C}^{n} \times \mathbb{R}$. Let

$$
\widetilde{\Gamma}_{m}(t, \widetilde{x}, \widetilde{y}):=\frac{1}{2 \pi} \sum_{\gamma \in \Gamma} \sum_{j=1}^{N} \int_{-\pi}^{\pi} \widetilde{H}_{\gamma, j, m}\left(t, \widetilde{x}, e^{i u} \circ \widetilde{y}\right) e^{i m u} d u .
$$

Note that when $\Gamma=\{e\}, \widetilde{\Gamma}_{m}(t, \widetilde{x}, \widetilde{y})=\Gamma_{m}(t, \pi(\widetilde{x}), \pi(\widetilde{y}))$ is defined in [16, (3.31)].

From Lemma 3.4, off-diagonal estimates of $\widetilde{A}_{B_{j}, m}(t, \widetilde{x}, \widetilde{y})$ (see (3.19)), we can repeat the proof of Theorem 5.14 in [8] with minor change and deduce that, see [19, Theorem 3.9],

Theorem 3.8. For every $\ell \in \mathbb{N}, \ell \geq 2$, and every $M>0$, there are $\epsilon_{0}>0$ and $m_{0}>0$ independent of $t$ and $m$ such that for every $m \geq m_{0}$, we have

$$
\left\|e^{-\frac{t}{m} \widetilde{\square}_{b, m}}(\widetilde{x}, \widetilde{y})-\widetilde{\Gamma}_{m}(t, \widetilde{x}, \widetilde{y})\right\|_{C^{l}(\widetilde{X} \times \widetilde{X})} \leq e^{-\frac{m}{t} \epsilon_{0}}, \quad \forall t \in(0, M) .
$$

From Theorem 3.6.4 in [23], we have

Proposition 3.9. For any $t_{0}>0, \varepsilon>0$ and any $\gamma \in \Gamma, j=1,2, \cdots, N$, there exists $C>0$ such that for any $z \in \widetilde{U}_{\gamma, j}, m \in \mathbb{N}, t>t_{0}$,

$$
\left\|\widetilde{A}_{B_{\gamma, j}, m}(t, z, z)-A_{B_{j}, m}(t, \pi(z), \pi(z))\right\|_{C^{l}\left(\widetilde{U}_{\gamma, j} \times \widetilde{U}_{\gamma, j}\right)} \leq C \exp \left(-\frac{m}{32 t} \varepsilon\right) .
$$

From (3.11) of [19] (see also (3.31) in [16]), (3.25), (3.26), Proposition 3.9 and the fact that $\widetilde{\psi}_{\gamma, j}=\psi_{j} \circ \pi$, we can easily deduce that

Lemma 3.10. With the above notations and assumptions as in Theorem 3.8 , we have

$$
\left\|\widetilde{\Gamma}_{m}(t, \widetilde{x}, \widetilde{x})-\Gamma_{m}(t, \pi(\widetilde{x}), \pi(\widetilde{x}))\right\|_{C^{l}(\widetilde{X} \times \widetilde{X})} \leq C \exp \left(-\frac{m}{t} \epsilon_{0}\right) .
$$


From Theorem 3.8, Lemma 3.10 and Theorem 3.5 of [16], we have, 19, Theorem 3.12],

Theorem 3.11. For every $\ell \in \mathbb{N}, \ell \geq 2$, and every $M>0$, there are $\epsilon_{0}>0$ and $m_{0}>0$ independent of $t$ and $m$ such that for any $\widetilde{x} \in \widetilde{X}$ and $m \geq m_{0}$, we have

$\left\|e^{-\frac{t}{m} \widetilde{\square}_{b, m}}(\widetilde{x}, \widetilde{x})-e^{-\frac{t}{m} \square_{b, m}}(\pi(\widetilde{x}), \pi(\widetilde{x}))\right\|_{C^{l}(\widetilde{X} \times \widetilde{X})} \leq C \exp \left(-\frac{m}{t} \epsilon_{0}\right), \forall t \in(0, M)$.

By Theorem 3.2 and Theorem 3.11, we have, 19, Theorem 3.13],

Theorem 3.12. With the above notations and assumptions, for every $\epsilon>0$, there are $m_{0}>0, \varepsilon_{0}>0$ and $C>0$ such that for all $m \geq m_{0}$, we have

$$
\begin{aligned}
& \left|e^{-\frac{t}{m} \widetilde{\square}_{b, m}}(\widetilde{x}, \widetilde{x})-\sum_{s=1}^{p} e^{\frac{2 \pi(s-1)}{p} m i}(2 \pi)^{-n-1} m^{n} \frac{\operatorname{det}(\dot{\mathcal{R}}) \exp \left(t \gamma_{d}\right)}{\operatorname{det}(1-\exp (-t \dot{\mathcal{R}}))}(\pi(\widetilde{x})) \otimes \operatorname{Id}_{E_{\pi(\widetilde{x})}}\right| \\
& \leq \epsilon m^{n}+C m^{n} t^{-n} e^{\frac{-\varepsilon_{0} m \hat{d}\left(\pi(\widetilde{x}), X_{\text {sing }}\right)^{2}}{t}}, \forall(t, \widetilde{x}) \in \mathbb{R}_{+} \times \widetilde{X}_{\text {reg }} .
\end{aligned}
$$

Recall that since $\Gamma$ acts on $\widetilde{X}$ freely so that $\widetilde{X} / \Gamma=X$, hence, we have $\tilde{X}_{\text {reg }} / \Gamma=X_{\text {reg }}$.

\subsection{Asymptotic expansion for the heat kernels of the Kohn Lapla-} cians

From Theorem 3.11 and Theorem 3.6 of [16], we have

Theorem 3.13. There exist $A_{m, \ell}(t, \pi(\widetilde{x})) \in C^{\infty}\left(\mathbb{R}_{+} \times X, \operatorname{End}\left(T^{* 0, \bullet} X \otimes E\right)\right)$ with $\left|A_{m, \ell}(t, \pi(\widetilde{x}))\right| \leq C_{\ell}$, for every $(t, \widetilde{x}) \in \mathbb{R}_{+} \times \widetilde{X}$, where $C_{\ell}>0$ is a constant independent of $m, \ell=-n,-n+1, \ldots$, such that for any $k \in \mathbb{N}$, there exists $C>0$ such that for any $t \in] 0,1], m \in \mathbb{N}^{*}$ and every $\widetilde{x} \in \widetilde{X}$, we have the asymptotic expansion

$$
\left|m^{-n} e^{-\frac{t}{m} \widetilde{\square}_{b, m}}(\widetilde{x}, \widetilde{x})-\sum_{\ell=-n}^{k} A_{m, \ell}(t, \pi(\widetilde{x})) t^{\ell}\right| \leq C t^{k},
$$

Moreover, for every $\ell=-n,-n+1, \ldots$, we can find $A_{m, \ell}(\pi(\widetilde{x})) \in C^{\infty}(X$, 
$\left.\operatorname{End}\left(T_{\pi(\widetilde{x})}^{* 0, \bullet} X \otimes E_{\pi(\widetilde{x})}\right)\right)$ with

$$
A_{m, \ell}(\pi(\widetilde{x}))=A_{\ell}(\pi(\widetilde{x})) \otimes \operatorname{Id}_{E_{\pi(\widetilde{x})}}+O\left(m^{-1 / 2}\right)
$$

in $C^{0}\left(X, \operatorname{End}\left(T^{* 0, \bullet} X\right) \otimes E\right)$ uniformly on $X$ such that there exist $C>0$ and $\varepsilon_{0}>0$ independent of $t$ and $m$ such that for any $\left.\left.t \in\right] 0,1\right], m \in \mathbb{N}^{*}$, we have

$$
\left|A_{m, \ell}(t, \pi(\widetilde{x}))-A_{m, \ell}(\pi(\widetilde{x}))\right| \leq C e^{-\frac{\varepsilon_{0} m d\left(\pi(\widetilde{x}), X_{\text {sing }}\right)^{2}}{t}}, \quad \forall \widetilde{x} \in \widetilde{X}_{\mathrm{reg}}
$$

where $A_{\ell}(\pi(\widetilde{x})) \in C^{\infty}\left(X, \operatorname{End}\left(T^{* 0, \bullet} X\right)\right), \ell=-n,-n+1, \ldots$, are as in $(3.12)$ of [16].

Let $\operatorname{Tr}_{q}\left[\exp \left(-\frac{t}{m} \square_{b, m}\right)\right]$ be the trace of the operator $\exp \left(-\frac{t}{m} \square_{b, m}\right)$ acting on $\Omega_{m}^{0, q}(X, E)$. It is well-known that (see Theorem 8.10 in [28])

$$
\operatorname{Tr}_{q}\left[\exp \left(-\frac{t}{m} \square_{b, m}\right)\right]=\int_{X} \operatorname{Tr}_{q}\left[\exp \left(-\frac{t}{m} \square_{b, m}\right)(x, x)\right] d v_{X}(x) .
$$

By [23, (3.6.7)] and [23, (3.6.8)], as in (3.32), see also [19, Proposition $4.2]$,

Proposition 3.14. We have

$$
\operatorname{Tr}_{\Gamma, q}\left[\exp \left(-\frac{t}{m} \widetilde{\square}_{b, m}\right)\right]=\int_{U} \operatorname{Tr}_{q}\left[e^{-\frac{t}{m} \widetilde{\square}_{b, m}}(\widetilde{x}, \widetilde{x})\right] d v_{\widetilde{X}}(\widetilde{x})
$$

Now, we fix $q=0,1,2, \ldots, n$. Let $A \in\left(T_{x}^{* 0, \bullet} X \otimes E_{x}\right) \otimes\left(T_{x}^{* 0, \bullet} X \otimes E_{x}\right)$. Let $e_{1}(x), \cdots, e_{d}(x)$ be an orthonormal frame of $T_{x}^{* 0, q} X \otimes E_{x}$. We write $\operatorname{Tr}^{(q)} A:=\sum_{j=1}^{d}\left\langle A e_{j} \mid e_{j}\right\rangle_{E}$. For the proof of our main result, we need to know the asymptotic behavior of

$$
\int_{X} \operatorname{Tr}^{(q)} A_{m, \ell}(t, x) d v_{X}(x), \quad \ell=-n,-n+1, \ldots
$$

Let $U$ be a fundamental domain of $\pi: \widetilde{X} \rightarrow X$. Then we have

$$
\int_{U} \operatorname{Tr}_{q}\left[e^{-\frac{t}{m} \widetilde{\square}_{b, m}}(\widetilde{x}, \widetilde{x})\right] d v_{\widetilde{X}}(\widetilde{x})=\int_{X} \operatorname{Tr}_{q}\left[e^{-\frac{t}{m} \square_{b, m}}(x, x)\right] d v_{X}(x) .
$$

Then we have the following theorem. 
Theorem 3.15. With the notations used in Theorem 3.13, fix $\ell=-n,-n+$ $1, \ldots$ We can find $a_{m, \ell}^{\frac{j}{2}} \in \mathbb{R}, j=0,1,2, \ldots$, with

$$
\begin{aligned}
& \lim _{m \rightarrow \infty} a_{m, \ell}^{0}=\int_{X} \operatorname{Tr}_{q}\left(A_{\ell}(x) \otimes \operatorname{Id}_{E_{x}}\right) d v_{X}(x), \\
& \lim _{m \rightarrow \infty} a_{m, \ell}^{\frac{j}{2}}=0, \quad j=1,2, \ldots,
\end{aligned}
$$

$a_{m, \ell}^{\frac{j}{2}}$ is independent of $t$, for each $j,\left|a_{m, \ell}^{\frac{j}{2}}\right| \leq C_{j}$, for every $m \in \mathbb{N}$, where $C_{j}>0$ is a constant independent of $m, j=0,1,2, \ldots$, such that

$$
\int_{X} \operatorname{Tr}_{q} A_{m, \ell}(t, x) d v_{X}(x) \sim \sum_{j=0}^{\infty} a_{m, \ell^{\frac{j}{2}}}^{t^{\frac{j}{2}}} \text { as } t \rightarrow 0^{+}, \text {uniformly in } m .
$$

In particular,

$$
\begin{gathered}
m^{-n} \operatorname{Tr}_{\Gamma, q}\left[e^{\left.-\frac{t}{m} \widetilde{\square}_{b, m}\right] \sim} t^{-n} b_{m,-n}+t^{-n+\frac{1}{2}} b_{m,-n+\frac{1}{2}}+t^{-n+1} b_{-n+1}+t^{-n+\frac{3}{2}} b_{-n+\frac{3}{2}}\right. \\
+\cdots \text { as } t \rightarrow 0^{+}, \text {uniformly in } m,
\end{gathered}
$$

where for each $j=0, \frac{1}{2}, 1, \ldots, b_{m,-n+j} \in \mathbb{C}$ is independent of $t$ and there is a constant $\hat{C}_{j}>0$ independent of $m$, such that $\left|b_{m,-n+j}\right| \leq \hat{C}_{j}$, for every $m \in \mathbb{N}$, and

$\lim _{m \rightarrow \infty} b_{m,-n+\frac{j}{2}}=\int_{X} \operatorname{Tr}_{q}\left(A_{-n+\frac{j}{2}}(x) \otimes \operatorname{Id}_{E_{x}}\right) d v_{X}(x)$, if $j$ is an even number, $\lim _{m \rightarrow \infty} b_{m,-n+\frac{j}{2}}=0$, if $j$ is an odd number.

Proof. By (3.29), (3.33) and (3.34), using the proof of Theorem 3.7 of [16], we prove the theorem.

\subsection{Spectral gap of $\square_{b, m}^{(q)}$}

Fix $q=0,1, \ldots, n$. Let $\widetilde{\square}_{b, m}^{(q)}: \operatorname{Dom} \widetilde{\square}_{b, m}^{(q)} \subset L_{m}^{2}\left(\widetilde{X}, T^{* 0, q} \widetilde{X} \otimes \widetilde{E}\right) \rightarrow L_{m}^{2}(\widetilde{X}$, $\left.T^{* 0, q} \widetilde{X} \otimes \widetilde{E}\right)$ be the restriction of $\widetilde{\square}_{b, m}$ on $(0, q)$ forms. By the same argument as in Theorem 3.10 of [16], we have the following theorem.

Theorem 3.16. Let $\mu_{m}^{(q)}$ be the lowest eigenvalue of $\widetilde{\square}_{b, m}^{(q)}$. There exist constants $c_{1}>0, c_{2}>0$ not depending on $m$ such that, for $q \geq 1$ and $m \in \mathbb{N}$, 


$$
\mu_{m}^{(q)} \geq c_{1} m-c_{2}
$$

\section{Analytic torsion on CR manifolds with $S^{1}$-action}

In this section we first study Mellin transformation, then we define the Fourier components of the analytic torsion for a rigid CR vector bundle $E$ over the CR manifold $X$ with a transversal CR $S^{1}$-action.

\subsection{Mellin transformation}

Let $\Gamma(z)$ be the Gamma function on $\mathbb{C}$. Then for $\operatorname{Re} z>0$, we have

$$
\Gamma(z)=\int_{0}^{\infty} e^{-t} t^{z-1} d t
$$

$\Gamma(z)^{-1}$ is an entire function on $\mathbb{C}$ and

$$
\Gamma(z)^{-1}=z+O\left(z^{2}\right) \text { near } z=0 .
$$

We suppose that $f(t) \in C^{\infty}\left(\mathbb{R}_{+}\right)$verifies the following two conditions:

I.

$$
f(t) \sim \sum_{j=0}^{\infty} f_{-k+\frac{j}{2}} t^{-k+\frac{j}{2}} \text { as } t \rightarrow 0^{+},
$$

where $k \in \mathbb{N}_{0}, f_{-k+\frac{j}{2}} \in \mathbb{C}, j=0,1,2, \ldots$.

II. For every $\delta>0$, there exist $c>0, C>0$ such that

$$
|f(t)| \leq C e^{-c t}, \quad \forall t \geq \delta .
$$

Definition 4.1. The Mellin transformation of $f$ is the function defined for $\operatorname{Re} z>k$,

$$
M[f](z)=\frac{1}{\Gamma(z)} \int_{0}^{\infty} f(t) t^{z-1} d t .
$$

We recall the following theorem, [16, Theorem 4.2]. 
Theorem 4.2. $M[f]$ extends to a meromorphic function on $\mathbb{C}$ with poles contained in

$$
\left\{\ell-\frac{j}{2} ; \ell, j \in \mathbb{Z}\right\}
$$

and its possible poles are simple. Moreover, $M[f]$ is holomorphic at 0 ,

$$
M[f](0)=f_{0}
$$

and

$$
\begin{aligned}
\frac{\partial M[f]}{\partial z}(0)= & \int_{0}^{1}\left(f(t)-\sum_{j=0}^{2 k} f_{-k+\frac{j}{2}} t^{-k+\frac{j}{2}}\right) \frac{1}{t} d t \\
& +\int_{1}^{\infty} f(t) \frac{1}{t} d t+\sum_{j=0}^{2 k-1} \frac{f_{-k+\frac{j}{2}}}{\frac{j}{2}-k}-\Gamma^{\prime}(1) f_{0} .
\end{aligned}
$$

\subsection{Definition of the Fourier components of the analytic torsion}

Let $N$ be the number operator on $T^{* 0, \bullet} \widetilde{X}$, i.e. $N$ acts on $T^{* 0, q} \widetilde{X}$ by multiplication by $q$. Fix $q=0,1, \cdots, n$ and take a point $\widetilde{x} \in \widetilde{X}$. Let $e_{1}(\widetilde{x}), \cdots, e_{d}(\widetilde{x})$ be an orthonormal frame of $T_{\widetilde{x}}^{* 0, q} \widetilde{X} \otimes \widetilde{E}_{\widetilde{x}}$. Let $A \in\left(T_{\widetilde{x}}^{* 0, \bullet} \widetilde{X} \otimes\right.$ $\left.\widetilde{E}_{\widetilde{x}}\right) \otimes\left(T_{\widetilde{x}}^{* 0, \bullet} \widetilde{X} \otimes \widetilde{E}_{\widetilde{x}}\right)$. Put $\operatorname{Tr}_{q} A:=\sum_{j=1}^{d}\left\langle A e_{j} \mid e_{j}\right\rangle_{\widetilde{E}}$ and set

$$
\begin{aligned}
\operatorname{Tr} A & :=\sum_{j=0}^{n} \operatorname{Tr}_{j} A, \\
\mathrm{~S} \operatorname{Tr} A & :=\sum_{j=0}^{n}(-1)^{j} \operatorname{Tr}_{j} A .
\end{aligned}
$$

Let $A: C^{\infty}\left(\widetilde{X}, T^{* 0, \bullet} \widetilde{X} \otimes \widetilde{E}\right) \rightarrow C^{\infty}\left(\widetilde{X}, T^{* 0, \bullet} \widetilde{X} \otimes \widetilde{E}\right)$ be a continuous operator with distribution kernel $A(\widetilde{x}, \widetilde{y}) \in C^{\infty}\left(\widetilde{X} \times \widetilde{X},\left(T_{\widetilde{y}}^{* 0, \bullet} \widetilde{X} \otimes \widetilde{E}_{\widetilde{y}}\right) \otimes\left(T_{\widetilde{x}}^{* 0, \bullet} \widetilde{X} \otimes \widetilde{E}_{\widetilde{x}}\right)\right)$. As in (3.33), we set

$$
\operatorname{Tr}_{\Gamma, q}[A]:=\int_{U} \operatorname{Tr}_{q} A(\widetilde{x}, \widetilde{x}) d v_{\widetilde{X}}(\widetilde{x})
$$


and put

$$
\begin{aligned}
\operatorname{Tr}_{\Gamma}[A] & :=\sum_{j=0}^{n} \operatorname{Tr}_{\Gamma, j}[A] \\
\operatorname{STr}_{\Gamma}[A] & :=\sum_{j=0}^{n}(-1)^{j} \operatorname{Tr}_{\Gamma, j}[A]
\end{aligned}
$$

Let

$$
\widetilde{\Pi}_{m}: L_{m}^{2}\left(\widetilde{X}, T^{* 0, \bullet} \widetilde{X} \otimes \widetilde{E}\right) \rightarrow \operatorname{Ker} \widetilde{\square}_{b, m}
$$

be the orthogonal projection and let

$$
\widetilde{\Pi}_{m}^{\perp}: L_{m}^{2}\left(\widetilde{X}, T^{* 0, \bullet} X \otimes \widetilde{E}\right) \rightarrow\left(\operatorname{Ker} \widetilde{\square}_{b, m}\right)^{\perp}
$$

be the orthogonal projection, where

$$
\left(\operatorname{Ker} \widetilde{\square}_{b, m}\right)^{\perp}=\left\{u \in L_{m}^{2}\left(\widetilde{X}, T^{* 0, \bullet} \widetilde{X} \otimes \widetilde{E}\right) ;(u \mid v)_{\widetilde{E}}=0, \quad \forall v \in \operatorname{Ker} \widetilde{\square}_{b, m}\right\} .
$$

By [8, Theorem 1.7], we have the following asymptotic expansion:

$$
\mathrm{S} \operatorname{Tr}\left[N e^{-t \square_{b, m}}\right] \sim \sum_{j=0}^{\infty} \hat{B}_{m,-n+\frac{j}{2}} t^{-n+\frac{j}{2}} \text { as } t \rightarrow 0^{+},
$$

where $\hat{B}_{m,-n+\frac{j}{2}} \in \mathbb{C}$ is independent of $t, j=0,1,2, \ldots$

By (3.34) and Lemma 4.3 of [16], we have the following

Lemma 4.3. Fix $q=0,1, \ldots, n$. For every $\delta>0$, there exist $c>0, C>0$ such that

$$
\left|\operatorname{Tr}_{\Gamma, \mathrm{q}}\left[e^{-t \widetilde{\square}_{b, m}} \Pi_{m}^{\perp}\right]\right| \leq C e^{-c t}, \quad \forall t \geq \delta
$$

From (3.34), (4.9) and Lemma 4.3, we see that $\operatorname{STr}_{\Gamma}\left[N e^{-t \widetilde{\square}_{b, m} \Pi \Pi_{m}^{\perp}}\right]$ satisfies (4.2) and (4.3). By Definition 4.1, for $\operatorname{Re}(z)>n$, we can define

$$
\widetilde{\theta}_{b, m}(z)=-M\left[\operatorname{STr}_{\Gamma}\left[N e^{-t \widetilde{\square}_{b, m}} \Pi_{m}^{\perp}\right]\right]=-\operatorname{STr}_{\Gamma}\left[N\left(\widetilde{\square}_{b, m}\right)^{-z} \Pi_{m}^{\perp}\right] .
$$

By Theorem 4.2, we have the following lemma. 
Lemma 4.4. $\widetilde{\theta}_{b, m}(z)$ extends to a meromorphic function on $\mathbb{C}$ with poles contained in the set

$$
\left\{\ell-\frac{j}{2} ; \ell, j \in \mathbb{Z}\right\}
$$

its possible poles are simple, and $\widetilde{\theta}_{b, m}(z)$ is holomorphic at 0 . Moreover,

$$
\begin{aligned}
\widetilde{\theta}_{b, m}^{\prime}(0)= & -\int_{0}^{1}\left\{\operatorname{STr}_{\Gamma}\left[N e^{-t \widetilde{\square}_{b, m}}\right]-\sum_{j=0}^{2 n} \hat{B}_{m,-n+\frac{j}{2}} t^{-n+\frac{j}{2}}\right\} \frac{d t}{t} \\
& -\int_{1}^{\infty} \operatorname{STr}_{\Gamma}\left[N e^{-t \widetilde{\square}_{b, m}} \Pi_{m}^{\perp}\right] \frac{d t}{t}-\sum_{j=0}^{2 n-1} \hat{B}_{m,-n+\frac{j}{2}} \frac{1}{\frac{j}{2}-n} \\
& +\Gamma^{\prime}(1)\left(\hat{B}_{m, 0}-\operatorname{STr}_{\Gamma}\left[N \Pi_{m}\right]\right) .
\end{aligned}
$$

Definition 4.5. Fix $m \in \mathbb{Z}$. We define $\exp \left(-\frac{1}{2} \widetilde{\theta}_{b, m}^{\prime}(0)\right)$ as the $m$-th Fourier component of the $L^{2}$-analytic torsion for the rigid vector bundle $\widetilde{E}$ over the CR covering manifold $\widetilde{X}$ with a transversal CR $S^{1}$-action.

\section{The Asymptotics of the Analytic Torsion}

Recall that we work with the assumption that $X$ is strongly pseudoconvex. From Theorem 3.15, we deduce

Theorem 5.1. With the notations used before, we have

$$
m^{-n} \operatorname{STr} \Gamma\left[N e^{-\frac{t}{m} \widetilde{\square}_{b, m}}\right] \sim \sum_{j=0}^{\infty} B_{m,-n+\frac{j}{2}} t^{-n+\frac{j}{2}} \text { as } t \rightarrow 0^{+} \text {, uniformly in } m,
$$

where, for each $j=0, \frac{1}{2}, 1, \ldots, B_{m,-n+j} \in \mathbb{C}$ is independent of $t$ and there is a constant $\hat{C}_{j}>0$ independent of $m$, such that $\left|B_{m,-n+j}\right| \leq \hat{C}_{j}$, for every $m \in \mathbb{N}$, and

$$
\begin{aligned}
& \lim _{m \rightarrow \infty} B_{m,-n+\frac{j}{2}}=\operatorname{rk}(E) \int_{X} \operatorname{STr}_{\Gamma} N A_{-n+\frac{j}{2}}(x) d v_{X}, \text { if } j \text { is an even number, } \\
& \lim _{m \rightarrow \infty} B_{m,-n+\frac{j}{2}}=0, \quad \text { if } j \text { is an odd number, }
\end{aligned}
$$

where $A_{\ell}(x) \in C^{\infty}\left(X\right.$, End $\left.\left(T^{* 0, \bullet} X\right) \otimes E\right)$ is as in $(3.12)$ of [16], $\ell=-n,-n+$ $1, \ldots$ 


\subsection{Asymptotics of the analytic torsion}

By Theorem 3.16 and Theorem 3.12, using the same proof of Theorem 3.10 of [16], we have

Theorem 5.2. There exist $C, c, c^{\prime}>0$ such that for any $q \geq 1, t \geq 1, m \in \mathbb{N}$, we have

$$
m^{-n} \operatorname{Tr}_{\Gamma, q}\left[e^{-\frac{t}{m} \widetilde{\square}_{b, m}}\right] \leq C \exp \left(-\left(c-c^{\prime} / m\right) t\right) .
$$

By the above results and proceeding as in the proof of Theorem 5.5 of [16], we have the main result of this work

Theorem 5.3. As $m \rightarrow+\infty$, we have

$$
\begin{aligned}
& \widetilde{\theta}_{b, m}^{\prime}(0)=\frac{p r}{4 \pi} \int_{X} \log \left(\operatorname{det}\left(\frac{m \dot{\mathcal{R}}}{2 \pi}\right)\right) e^{-m \frac{d \omega_{0}}{2 \pi}} \wedge\left(-\omega_{0}\right)+o\left(m^{n}\right), \text { for } p \mid m, \\
& \widetilde{\theta}_{b, m}^{\prime}(0)=o\left(m^{n}\right), \quad \text { for } \quad p \nmid m,
\end{aligned}
$$

where $r$ denotes the rank of $\widetilde{E}$ and $\dot{\mathcal{R}} \in \operatorname{End}\left(T^{1,0} X\right)$ is defined in (3.6).

\section{References}

1. M.-S. Baouendi and L.-P. Rothschild and F.-Treves, CR structures with group action and extendability of CR functions, Invent. Math., 83 (1985), 359-396.

2. J.-M. Bismut, Demailly's asymptotic Morse inequalities: a heat equation proof, J. Funct. Anal., 72 (1987), 263-278.

3. J.-M. Bismut and E. Vasserot, The asymptotics of the Ray-Singer analytic torsion associated with high powers of a positive line bundle, Comm. Math. Phys., 125, (1989), no.2, 355-367.

4. M. Braverman, A. Carey, M. Farber and V. Mathai, $L^{2}$ torsion without the determinant class condition and extended $L^{2}$ cohomology, Commun. Contemp. Math. 7 (2005), no. 4, 421-462.

5. D. Burghelea, L. Friedlander, T. Kappeler, and P. McDonald, Analytic and Reidemeister torsion for representations in finite type Hilbert modules, Geom. Funct. Anal., 6 (1996), no. 5, 751-859.

6. A. Carey, M. Farber, and V. Mathai, Correspondences, von Neumann algebras and holomorphic $L^{2}$ torsion, Canad. J. Math., 52 (2000), no. 4, 695-736.

7. A. Carey and V. Mathai, $L^{2}$-torsion invariants, J. Funct. Anal., 110 (1992), no. 2, 377-409. 
8. J.-H. Cheng, C.-Y. Hsiao and I.-H. Tsai, Heat kernel asymptotics and a local index theorem for CR manifolds with $S^{1}$ action, Mém. Soc. Math. Fr. (N.S.) no. 162 (2019), vi+139 pp.

9. J. P. Demailly, Champs magnétiques et inégalités de Morse pour la $d$ "-cohomologie, Ann. Inst. Fourier, 35 (1985), 189-229.

10. J.-J. Duistermaat and G.-J. Heckman, On the Variation in the Cohomology of the Sympleetic Form of the Reduced Phase Space, Invent. Math., 69(1982), 259-268.

11. M. Farber, von Neumann categories and extended L2-cohomology, K-Theory, 15 (1998), no. 4, 347-405.

12. S. Finski, On the full asymptotics of analytic torsion, J. Funct. Anal., 275 (2018), no. $12,3457-3503$.

13. Alexander Grigoryan and Laurent Saloff-Coste, Dirichlet heat kernel in the exterior of a compact set, Comm. Pure Appl. Math., 55(2002), 93-133.

14. M. Gromov, M. G. Henkin, M. Shubin, $L^{2}$ holomorphic functions on pseudo-convex coverings, GAFA, 8 (1998), 552-585.

15. C.-Y. Hsiao, Szegö kernel asymptotics for high power of CR line bundles and Kodaira embedding theorems on CR manifolds, Mem. Amer. Math. Soc., 254 (2018), no. 1217, $\mathrm{v}+142 \mathrm{pp}$.

16. C.-Y. Hsiao and R.-T. Huang, The asymptotics of the analytic torsion on CR manifolds with $S^{1}$-action, Comm. Contem. Math., 21 (2019), no. 4, 1750094, 35 pp.

17. C.-Y. Hsiao and X. Li, Szegö kernel asymptotics and Morse inequalities on CR manifolds with $S^{1}$ action, Asian J. Math., 22 (2018), no. 3, 413-450.

18. C.-Y. Hsiao and Xiaoshan Li, Morse inequalities for Fourier components of Kohn-Rossi cohomology of CR manifolds with $S^{1}$-action, Math. Z., 284 (2016), no. 1-2, 441-468.

19. R.-T. Huang and G. Shao, Morse inequalities for Fourier components of Kohn-Rossi cohomology of CR covering manifolds with $S^{1}$-action, to appear in Pacific J. Math., arXiv:1809.06243.

20. T. Kawasaki, The Riemann-Roch theorem for complex V-manifolds, Osaka J. Math., 16(1)(1979), 151-159.

21. J. Lott, Heat kernels on covering spaces and topological invariants, J. Differential Geom., 35 (1992), no. 2, 471-510.

22. X. Ma, Orbifolds and analytic torsion, Trans. Amer. Math. Soc., 357 (2005), no. 6, 2205-2233.

23. X. Ma and G. Marinescu, Holomorphic Morse inequalities and Bergman kernels, Progress in Mathematics, 254, Birkhäuser Verlag, Basel, (2007).

24. V. Mathai, $L^{2}$-analytic torsion, J. Funct. Anal., 107 (1992), no. 2, 306-386.

25. L. Ornea and M. Verbitsky, Sasakian structures on CR-manifolds, Geometriae Dedicata, 125(1), (2007), 159-173. 
"BN14N43" — 2019/12/24 — 9:26 — page 491 — \#31

26. P-E. Paradan and M. Vergne, Index of transversally elliptic operators, Astérisque, No. 328 (2009), 297-338.

27. M. Puchol, The asymptotic of the holomorphic analytic torsion forms, ArXiv: 1511.04694v1.

28. J. Roe, Elliptic Operators, Topology and Asymptotic Methods, Second edition, Pitman Research Notes in Mathematics Series, 395, Longman, Harlow, (1998).

29. D. B. Ray and I. M. Singer, R-torsion and the Laplacian on Riemannian Manifolds, Adv. in Math., 7 (1971), 145-210.

30. D. B. Ray and I. M. Singer, Analytic torsion for complex manifolds, Ann. of Math., 98 (1973), no. 2 ,154-177.

31. Y. T. Siu, A vanishing theorem for semipositive line bundles over non-Kähler manifolds, J. Diff. Geom., 19 (1984), 431-452.

32. G. Su, Holomorphic $L^{2}$ torsion without determinant class condition, Proc. Amer. Math. Soc., 143 (2015), no. 10, 4513-4524.

33. R. Todor, I. Chiose and G. Marinescu, Morse inequalities for covering manifolds, Nagoya Math. J., 163 (2001), 145-165.

34. W. Zhang, An extended Cheeger-Müller theorem for covering spaces, Topology, 44 (2005), no. 6, 1093-1131. 